\title{
The distributed complexity of locally checkable problems on paths is decidable
}

\author{
Alkida Balliu · alkida.balliu@aalto.fi · Aalto University \\ Sebastian Brandt · brandts@ethz.ch · ETH Zurich \\ Yi-Jun Chang · cyijun@umich.edu · University of Michigan \\ Dennis Olivetti · dennis.olivetti@aalto.fi · Aalto University \\ Mikaël Rabie · mikael.rabie@irif.fr · Aalto University and IRIF, University Paris Diderot \\ Jukka Suomela · jukka.suomela@aalto.fi · Aalto University
}

\begin{abstract}
Consider a computer network that consists of a path with $n$ nodes. The nodes are labeled with inputs from a constant-sized set, and the task is to find output labels from a constant-sized set subject to some local constraints-more formally, we have an LCL (locally checkable labeling) problem. How many communication rounds are needed (in the standard LOCAL model of computing) to solve this problem?

It is well known that the answer is always either $O(1)$ rounds, or $\Theta\left(\log ^{*} n\right)$ rounds, or $\Theta(n)$ rounds. In this work we show that this question is decidable (albeit PSPACEhard): we present an algorithm that, given any LCL problem defined on a path, outputs the distributed computational complexity of this problem and the corresponding asymptotically optimal algorithm.
\end{abstract}




\section{Introduction}

To what extent is it possible to automate the design of algorithms and the study of computational complexity? While algorithm synthesis problems are typically undecidable, there are areas of theoretical computer science in which we can make use of computational techniques in algorithm design - at least in principle, and sometimes also in practice. One such area is the theory of distributed computing; see [3, 5, 6, 9, 11, 17, 19, 26] for examples of recent success stories. In this work we bring yet another piece of good news:

Consider this setting: there is a computer network that consists of a path with $n$ nodes, the nodes are labeled with inputs from a constant-sized set, and the task is to find output labels from a constant-sized set subject to some local constraints. We show that for any given set of local constraints, it is decidable to tell what is the asymptotically optimal number of communication rounds needed to solve this problem (as a function of $n$, for the worst-case input).

Background: LCLs and the LOCAL Model. We focus on what are known as LCL (locally checkable labeling) problems [22] in the LOCAL model of distributed computing [20, 24]. We define the setting formally in Section 2, but in essence we look at the following question:

- We are given an unknown input graph of maximum degree $\Delta=O(1)$; the nodes are labeled with input labels from a constant-size set $\Sigma_{\text {in }}$, and the nodes also have unique identifiers from a polynomially-sized set.

- The task is to label the nodes with output labels from a constant-size set $\Sigma_{\text {out }}$, subject to some local constraints $\mathcal{P}$; a labeling is globally feasible if it is locally feasible in all radius- $r$ neighborhoods for some $r=O(1)$.

- Each node has to produce its own output label based on the information that it sees in its own radius- $T(n)$ neighborhoods for some function $T$.

Here the local constraints $\mathcal{P}$ define an $\mathrm{LCL}$ problem. The rule that the nodes apply to determine their output labels is called a distributed algorithm in the LOCAL model, and function $T(n)$ is the running time of the algorithm-here $T(n)$ determines how far a node has to see in order to choose its own part of the solution, or equivalently, how many communication rounds are needed for each node to gather the relevant information if we view the input graph as a communication network.

In this setting, the case of $T(n)=\Theta(n)$ is trivial, as all nodes can see the entire input. The key question is to determine which problems $\mathcal{P}$ can be solved in sublinear time-here are some examples:

- Vertex coloring with $\Delta+1$ colors: can be solved in time $O\left(\log ^{*} n\right)[8,16]$ and this is tight $[20,21]$.

- Vertex coloring with $\Delta$ colors, for $\Delta>2$ : can be solved in polylogarithmic time [23] and requires at least logarithmic time [7] for deterministic algorithms.

While the study of this setting was initiated already in the seminal work by Naor and Stockmeyer in 1995 [22], our understanding of these questions has rapidly advanced in the past three years $[1,2,4,6,7,12-15,25]$. The big surprises have been these: 
- There are LCL problems with infinitely many different time complexities - for example, we can construct $L C L$ problems with a time complexity exactly $\Theta\left(n^{\alpha}\right)$ for any rational number $0<\alpha \leq 1$.

- Nevertheless, there are also wide gaps in the complexity landscape: for example, no LCL problem has a (deterministic) computational complexity that is between $\omega\left(\log ^{*} n\right)$ and $o(\log n)$.

However, what is perhaps most relevant for us is the following observation: if we look at the case of $\Delta=2$ (paths and cycles), then the time complexity of any LCL problem is either $O(1), \Theta\left(\log ^{*} n\right)$, or $\Theta(n)$, and the same holds for both deterministic and randomized algorithms $[5,6,22]$.

Decidability of LCL Time Complexities. For a fixed $\Delta$, any LCL problem has a trivial finite representation: simply enumerate all feasible radius- $r$ local neighborhoods. Hence it makes sense to ask whether, given an LCL problem, it is possible to determine its time complexity. The following results are known by prior work:

- If the input graph is an unlabeled path or cycle, the time complexity is decidable $[5,22]$.

- If the input graph is a grid or toroidal grid, the time complexity is undecidable [22]. However, there are also some good news: in unlabeled toroidal grids, the time complexity falls in one of the classes $O(1), \Theta\left(\log ^{*} n\right)$, or $\Theta(n)$, it is trivial to tell if the time complexity is $O(1)$, and it is semi-decidable to tell if it is $\Theta\left(\log ^{*} n\right)$ [5].

- In the case of trees, there are infinitely many different time complexities, but there is a gap between $\omega(\log n)$ and $n^{o(1)}$, and it is decidable to tell on which side of the gap a given problem lies $[6]$.

Somewhat surprisingly, the seemingly simple case of labeled paths or cycles has remained open all the way since the 1995 paper by Naor and Stockmeyer [22], which defined LCLs with inputs but analyzed decidability questions only in the case of unlabeled graphs.

We initially expected that the question of paths with input labels is a mere technicality and the interesting open questions are related to much broader graph families, such as rooted trees, trees, and bounded-treewidth graphs. However, it turned out that the main obstacle for understanding decidability in any such graph family seems to lie in the fact that the structure of the graph can be used to encode arbitrary input labels, hence it is necessary to first understand how the input labels influence decidability - and it turns out that this makes all the difference in the case of paths.

In this work we show that the time complexity of a given LCL problem on labeled paths or cycles is decidable. However, we also show that decidability is far from trivial: the problem is PSPACE-hard, as LCL problems on labeled paths are expressive enough to capture linear bounded automata (Turing machines with bounded tapes).

\section{Model}

The LOCAL Model. The model of computation we consider in this work is the LOCAL model of distributed computing [20,24]. In the LOCAL model, each node of the input graph is considered as a computational entity that can communicate with the neighboring nodes in order to solve some given graph problem. Computation is divided into synchronous rounds, where in each round each node first sends messages of arbitrary size to its neighbors, then receives the messages sent by its neighbors, and finally performs some local computation of arbitrary complexity. Each node is equipped with a globally unique identifier (ID) which is simply a bit string of length $O(\log n)$, where 
$n$ denotes the number of nodes of the input graph. In the beginning of the computation, each node is aware of its own ID, the number of nodes and the maximum degree $\Delta$ of the input graph, and potentially some additional problem-specific input. Each node has to decide at some point that it terminates, upon which it returns a local output and does not take part in any further computation; the problem is solved correctly if the local outputs of all nodes together constitute a global output that satisfies the output constraints of the given problem.

Each node executes the same algorithm; the running time of the distributed algorithm is the number of rounds until the last node terminates. It is well known that, due to the unbounded message sizes, an algorithm with runtime $T(n)$ can be equivalently described as a function from the set of all possible radius- $T(n)$ neighborhoods to the set of allowed outputs. In other words, we can assume that in a $T(n)$-round algorithm, each node first gathers the topology of and the input labels contained in its radius- $T(n)$ neighborhood, and then decides on its output based solely on the collected information.

Locally Checkable Labelings. The class of problems we consider is locally checkable labeling ( $\mathrm{LCL}$ ) problems [22]. LCL problems are defined on graphs of bounded degree, i.e., we will assume that $\Delta=O(1)$. Formally, an $\mathrm{LCL}$ problem is given by a finite input label set $\Sigma_{\text {in }}$, a finite output label set $\Sigma_{\text {out }}$, an integer $r$, and a finite set $\mathcal{C}$ of graphs where every node is labeled with a pair $\left(\ell_{\text {in }}, \ell_{\text {out }}\right) \in \Sigma_{\text {in }} \times \Sigma_{\text {out }}$ and one node is marked (as the center). Each node of the input graph is assigned an input label from $\Sigma_{\text {in }}$ before the computation begins, and the global output of a distributed algorithm is correct if the radius- $r$ neighborhood of each node $v$, including the input labels given to the contained nodes and the output labels returned by the contained nodes, is isomorphic to an element of $\mathcal{C}$ where $v$ corresponds to the node marked as the center.

In the case of directed paths as our class of input graphs, we are interested in identifying the simplest possible form of LCL problems. For this purpose, we define $\beta$-normalized LCLs; these are problems for which the input is just binary, and the size of the set of output labels is $\beta$. Moreover, the solution can be checked at each node $v$ by just inspecting the input and output of $v$, and, separately, the output of $v$ and the output of its predecessor. More formally, a $\beta$-normalized LCL problem is given by finite input and output label sets $\Sigma_{\text {in }}, \Sigma_{\text {out }}$ satisfying $\left|\Sigma_{\text {in }}\right|=2,\left|\Sigma_{\text {out }}\right|=\beta$, a finite set $\mathcal{C}_{\text {in - out }}$ of pairs $\left(\ell_{\text {in }}, \ell_{\text {out }}\right) \in \Sigma_{\text {in }} \times \Sigma_{\text {out }}$ and a finite set $\mathcal{C}_{\text {out - out }}$ of pairs $\left(\ell_{\text {out }}, \ell_{\text {out }}^{\prime}\right) \in \Sigma_{\text {out }} \times \Sigma_{\text {out }}$. The global output of a distributed algorithm for the $\beta$-normalized LCL problem is correct if the following hold:

- For each node $v$, we have $(\operatorname{Input}(v), \operatorname{Output}(v)) \in \mathcal{C}_{\text {in }- \text { out }}$, where $\operatorname{Input}(v)$ denotes the input label of $v$, and Output $(v)$ the output label of $v$.

- For each node $v$ that has a predecessor, we have (Output $(v)$, Output $(u)) \in \mathcal{C}_{\text {out - out }}$, where $u$ is the predecessor of $v$, and $\operatorname{Output}(v)$, Output $(u)$ are the output labels of $v$ and $u$, respectively.

It is straightforward to check that a $\beta$-normalized $\mathrm{LCL}$ problem is indeed a special case of an $\mathrm{LCL}$ problem where $r=1$.

\section{Hardness}

In this section we study the hardness of determining the distributed complexity of LCLs on paths and cycles with input labels. More precisely, we start by proving the existence of a family $\Pi$ of LCL problems for consistently globally oriented paths, such that, given an LCL problem in $\Pi$, it is PSPACE-hard to decide if its distributed complexity is $O(1)$ or $\Theta(n)$. Our main result shows the following. 


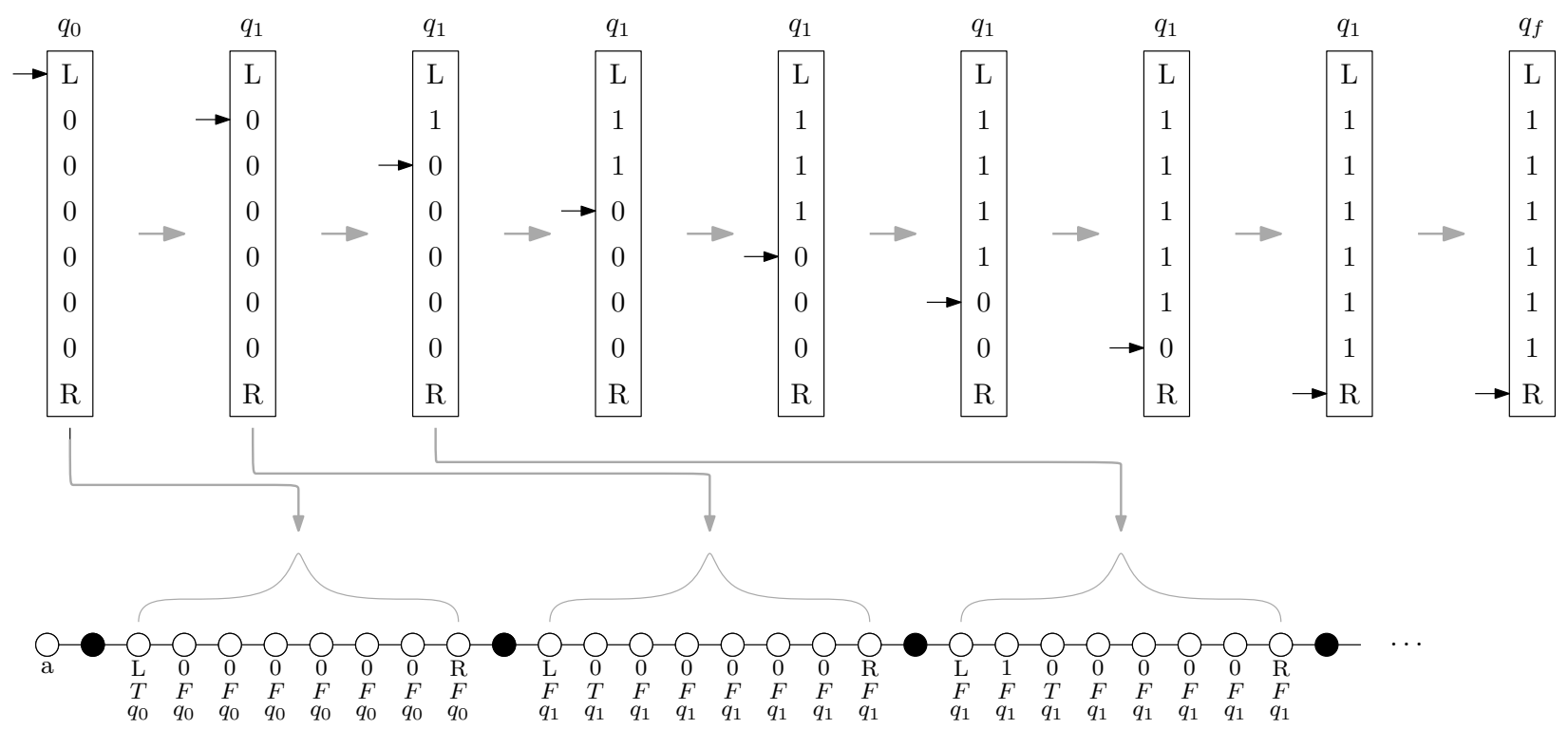

Figure 1: Illustration of a correct encoding of the execution of an LBA on a path; black nodes act as separators between the encoding of two consecutive steps of the LBA; in the example, the LBA executes a unary counter.

It is PSPACE-hard to distinguish whether a given $\mathrm{LCL}$ problem $\mathcal{P}$ with input labels can be solved in $O(1)$ time or needs $\Omega(n)$ time on globally oriented path graphs.

The high level idea of the proof of the above result is as follows. We would like to encode the execution of Turing machines as LCLs on consistently oriented paths, and then define some LCL for which the complexity depends on the running time of the machine. This is fairly easy on oriented grids, for example, where we can use one dimension of the grid as a tape, and the other dimension as time. One may try to do the same on paths, by projecting everything on a single dimension, concatenating the tape state of each step. Unfortunately, the obtained encoding is not locally checkable, since the length of the tape may be non-constant. Hence, in order to guarantee the local checkability, we should consider Turing machines having a tape of size at most $B$, where $B$ is a constant with respect to the number of nodes in the path where we want to encode its execution. For this purpose, we consider Linear Bounded Automata (LBA) [18, p. 225]. An LBA is a Turing machine that has a tape of size upper bounded by some $B$. We show that, if $B$ is constant with respect to the number of nodes in the path, we can then encode the execution of an LBA $M_{B}$ as an LCL for directed paths. Moreover, we show that by seeing this encoding as a two party game between a prover and a disprover, we can encode the execution of $M_{B}$ using labels of constant size that do not depend on $B$, even in the case in which the $\mathrm{LCL}$ checkability radius is 1 . If the execution of $M_{B}$ is not correctly encoded in the input of the $\mathrm{LCL}$, then we can disprove its correctness using output labels of size $O(B)$. Moreover, we ensure that, if the execution of $M_{B}$ is correctly encoded in the input of the $\mathrm{LCL}$, it is not possible to produce a correct proof of non-correctness. Then, in order to obtain an LCL with a distributed complexity that depends on the execution time of $M_{B}$, we encode some secret input at the first node of the path. We require then that all nodes involved in a correct encoding must produce the same secret as output.

Figure 1 shows an example of an LBA that executes a unary counter, and its encoding as input to nodes on a path. In this instance, all nodes must produce the symbol $a$ as output. Figure 2 shows an example of the wrong input (the tape has been copied incorrectly between two consecutive steps 


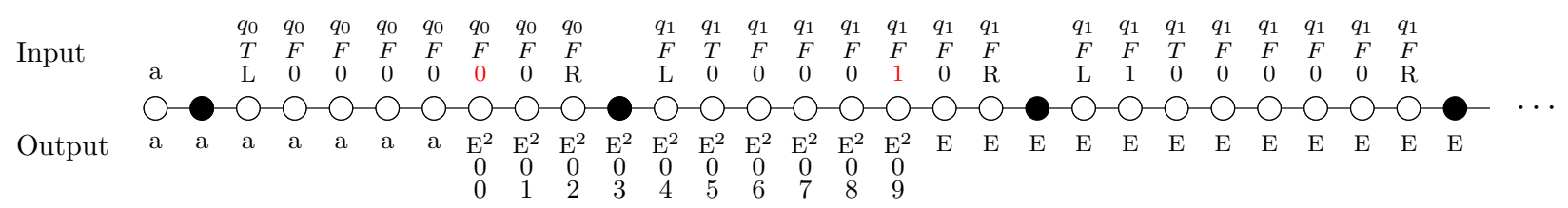

Figure 2: Illustration of an incorrect encoding of the execution of an LBA on a path; in the example, the tape of the LBA is wrongly copied (the inputs in red are different, while they should be the same). The error output $E^{2}$ encodes the distance of $B+1$ between the two nodes, and the input wrongly copied.

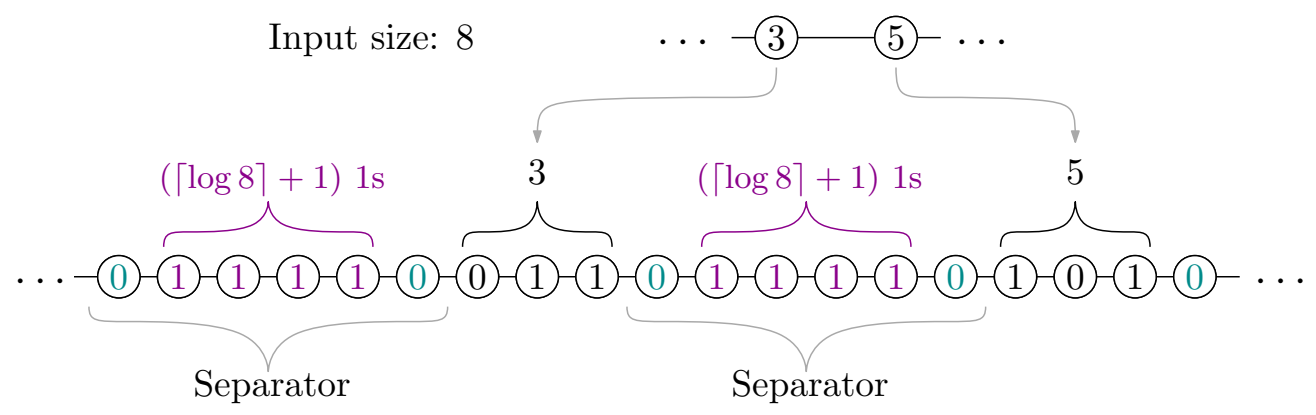

Figure 3: Illustration of the normalization of an LCL.

of the LBA). In this case, nodes are allowed to produce a chain of errors. Different types of errors will be handled using different types of error labels. In the example, all nodes that produce the error chain, output $E^{2}$, indicating an error of type 2 . We will show that we need $O(B)$ symbols to handle all possible errors (including the case in which the input tape is too long, way more than $B$ ). Also, it is necessary that all error chains that we allow as outputs must be locally checkable.

Another interesting problem is to identify, for an LCL that can be distributedly solved in constant time, how big this constant can be. In particular, we first focus on identifying the simplest possible description of an $\mathrm{LCL}$, and then, we provide a lower bound on the complexity of a constant time $\mathrm{LCL}$, as a function of the size of the $\mathrm{LCL}$ description. For this purpose, we consider $\beta$-normalized LCLs, i.e., problems for which the input labeling is just binary and there are $\beta$ possible output labels. Also, the verifier for these LCLs is the simplest possible: it can only check if the output of a node is correct w.r.t. its input, and separately, if the output of a node is correct w.r.t. the output of its predecessor. Therefore, we show how to convert an LCL to a $\beta$-normalized one by encoding the input in binary (Figure 3 shows an example), and obtain the following result.

There are $\beta$-normalized LCLs that can be solved in constant time but the distributed time complexity is $2^{\Omega(\beta)}$.

All results that we have been described so far apply to globally oriented paths. Nevertheless, we show that ideas and techniques can be generalized to work on undirected path and cycles as well, obtaining essentially the same results. Finally, we will show how to lift these results to trees without input labels, proving the following result.

It is PSPACE-hard to distinguish whether a given $\mathrm{LCL}$ problem $\mathcal{P}$ without input labels can be solved in $O(1)$ time or needs $\Omega(n)$ time on trees with degree $\Delta=3$. 


\subsection{Linear Bounded Automata}

A Linear Bounded Automata $M_{B}$ is a Turing Machine having a bounded tape of size at most $B$, such that it is able to recognize the boundaries of the tape [18, p. 225]. More formally, we define an LBA as a tuple of 5 elements $M=\left(Q, q_{0}, q_{f}, \Gamma, \delta\right)$, where

- $Q$ is a finite set of states;

- $q_{0} \in Q$ is the initial state;

- $q_{f} \in Q$ is the final state;

- $\Gamma$ is a finite set of tape alphabet symbols that contains integers 0,1 , and special symbols $L$ (left), and $R$ (right);

- $\delta$ is the transition function, where $\delta: Q \backslash\left\{q_{f}\right\} \times \Gamma \rightarrow Q \times \Gamma \times\{-, \leftarrow, \rightarrow\}$.

The tape of $M_{B}$ is initialized as follows:

- the first cell is marked with the symbol $L$;

- the last cell is marked with the symbol $R$;

- all other cells contain an integer in $\{0,1\}$.

An execution of an LBA is a sequence $\left(\operatorname{step}_{i} \mid i \in\{1, \ldots, t\}\right)$, where

- $\operatorname{step}_{i}=\left(\right.$ state $_{i}$, tape $_{i}$, head $\left._{i}\right)$;

- state $_{t}=q_{f}$;

- $\delta\left(\right.$ state $_{i}$, tape $\left._{i}\left[\operatorname{head}_{i}\right]\right)=\left(\right.$ state $\left._{i+1}, \operatorname{tape}_{i+1}\left[\operatorname{head}_{i}\right], \epsilon\right)$, and head $i+1$ is

$-\operatorname{head}_{i}-1$ if $\epsilon$ is $\leftarrow$;

- head $_{i}$ if $\epsilon$ is -;

- head $_{i}+1$ if $\epsilon$ is $\rightarrow$.

\subsection{The LCL Problem}

We define a family $\Pi$ of LCLs, in which each problem $\Pi_{M_{B}}$ depends on the LBA $M_{B}$. The general idea is that the input of the LCL may encode the execution of an LBA $M_{B}$. If it is the case, nodes are required to solve a problem that requires a time proportional to the execution time of $M_{B}$. On the other hand, if it is not the case, nodes can produce an output that proves that this encoding is wrong. In order to define valid LCLs, we consider the case where $B=O(1)$, that is, the size of the tape does not depend on the size of the distributed network.

\subsubsection{Input Labels}

We define the input labels of our LCL as follows:

- Start $(\phi)$, where $\phi \in\{a, b\}$, indicates a symbol that will be used as some kind of secret;

- Separator, a label that acts as a separator between two steps of $M_{B}$;

- Tape $(c, s, h)$ gives information about the tape and the state of $M_{B}$, where the content $c \in$ $\{0,1, L, R\}$, the state $s \in Q$, and the head $h \in\{$ true, false $\}$;

- Empty, indicating an empty input.

Note that the size of the set of possible input labels does not depend on the size $B$ of the tape. 


\subsubsection{Encoding an LBA on a Path}

Suppose we have a consistent global orientation in the path $P=\left(p_{0}, p_{1}, p_{2}, \ldots, p_{n-1}\right)$. Let step $=$ $\left(\right.$ step $_{i}=\left(\right.$ state $_{i}$, tape $_{i}$, head $\left.\left._{i}\right) \mid i \in\{1, \ldots, t\}\right)$ be the execution of the LBA $M_{B}$ starting from a tape initialized with $(L, 0, \ldots, 0, R)$.

Definition 1. The input of the LCL is a good input if the first node of the path has in input Start $(\phi)$, where $\phi \in\{a, b\}$, and the rest of the path correctly encodes the execution of an LBA $M_{B}$ initialized with $(L, 0, \ldots, 0, R)$ (see Figure 1$)$. More precisely:

- $\operatorname{Input}\left(p_{0}\right)=\operatorname{Start}(\phi)$

- Input $\left(p_{(i-1)(B+1)+1}\right)=$ Separator for $i \in 1, \ldots, t$;

- Input $\left(p_{(i-1)(B+1)+1+j}\right)=\operatorname{Tape}(c, s, h)$ for $i \in 1, \ldots, t, j \in\{1, \ldots, B\}$, where

$-c=\operatorname{tape}_{i}[j]$

$-s=$ state $_{i}$

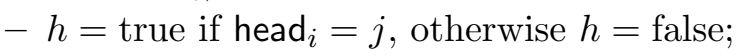

- All other nodes have in input Empty.

\subsubsection{Output Labels}

The set of output labels is the following.

- Start $(\phi)$

- Empty;

- Error: a generic error label;

- $\operatorname{Error}^{0}(i)$ where $0 \leq i \leq B+1$ : an error of type 0 indicating that the machine is not correctly initialized;

- $\operatorname{Error}^{1}(i)$, where $0 \leq i \leq B$ : an error of type 1 that we will use in the case where the size of the tape is not correct, i.e., when the size of the tape is not $B$;

- $\operatorname{Error}^{2}(x, i)$, where $x \in\{0,1, L, R\}$ and $0 \leq i \leq B+1$ : an error of type 2 used when the tape of $M_{B}$ is wrongly copied;

- Error ${ }^{3}$ : an error of type 3 is used in case nodes have inconsistent states;

- $\operatorname{Error}^{4}$ (current_state, tape_content, $i$ ), where $0 \leq i \leq B+2$ : an error of type 4 indicating that the transition of $M_{B}$ is encoded incorrectly (this error captures also the case where the head is missing);

- $\operatorname{Error}^{5}(x)$ where $x \in\{0,1\}$ : an error of type 5 used in the case when there is more than one head.

\subsubsection{LCL Constraints}

The high level idea is the following. If the path encodes a good input, then nodes that are not labeled Empty are required to output the input given to the first node of the path (either $a$ or $b$ ). Otherwise, nodes can produce a locally checkable proof of an error (see Figure 2 for an example). While nodes may output $a$ or $b$ even in the case in which the input is not a good input, nodes must not be able to produce a proof error in the case in which the path encodes a good input. We describe all these requirements as locally checkable constraints.

An output labeling for problem $\Pi_{M_{B}}$ is correct if the following conditions are satisfied for nodes of the path $P=\left(p_{0}, \ldots, p_{n-1}\right)$. Note that, although nodes do not know their position on the path, for the sake of simplicity we will denote with $p_{i-1}$ the predecessor of $p_{i}$, if it exists. 
1. Each node $v$ produces exactly one output label.

2. If Output $(v)=$ Empty then $\operatorname{Input}(v)=$ Empty.

3. If $v$ has no predecessors (i.e., $\left.v=p_{0}\right)$ and $\operatorname{Output}(v)=\operatorname{Start}(\phi)$, then $\operatorname{Input}(v)=\operatorname{Start}(\phi)$.

4. If Output $\left(p_{i-1}\right)=a$ then Output $\left(p_{i}\right) \neq b$, and if Output $\left(p_{i-1}\right)=b$ then $\operatorname{Output}\left(p_{i}\right) \neq a$.

5. If Output $\left(p_{i}\right)=\operatorname{Error}^{0}(j)$, then

- if $j=0$ then the node has no predecessor;

- if $j>0$ then Output $\left(p_{i-1}\right)=\operatorname{Error}^{0}(j-1)$.

6. If Output $\left(p_{i}\right)=\operatorname{Error}^{1}(j)$, then

- if $j=0$, then $\operatorname{Input}\left(p_{i}\right)=$ Separator;

- if $j>0$ then $\operatorname{Input}\left(p_{i}\right) \neq$ Separator and $\operatorname{Output}\left(p_{i-1}\right)=\operatorname{Error}^{1}(j-1)$.

7. If Output $\left(p_{i}\right)=\operatorname{Error}^{2}(x, j)$, then

- if $j=0$, then $\operatorname{Input}\left(p_{i}\right)=\operatorname{Tape}(c, s, h)$ where $h=$ false, $c=x$;

- if $j=B+1$ then $\operatorname{Input}\left(p_{i}\right)=\operatorname{Tape}(c, s, h)$ where $c \neq x$;

- if $0<j<B+1$ then $\operatorname{Output}\left(p_{i-1}\right)=\operatorname{Error}^{2}(x, j-1)$.

8. If Output $\left(p_{i}\right)=\operatorname{Error}^{3}$, then $\operatorname{Input}\left(p_{i}\right)=\operatorname{Tape}(c, s, h), \operatorname{Input}\left(p_{i-1}\right)=\operatorname{Tape}\left(c^{\prime}, s^{\prime}, h^{\prime}\right)$, and $s \neq s^{\prime}$.

9. If Output $\left(p_{i}\right)=\operatorname{Error}^{4}$ (current_state, tape_content, $j$ ), let (transition_state, new_content, $\epsilon$ ) $=$ $\delta$ (current_state, tape_content)

- if $j=0$, then $\operatorname{Input}\left(p_{i}\right)=\operatorname{Tape}(c, s, h)$ where $c=$ tape_content, $s=$ current_state, $h=$ true;

- if $j=B$ and $\epsilon=\leftarrow$, or $j=B+1$ and $\epsilon=-$, or $j=B+2$ and $\epsilon=\rightarrow$ (i.e., if node $p_{i}$ is an "Error ${ }^{4}$ final node"), then either current_state is a final state or $\operatorname{Input}\left(p_{i}\right)=\operatorname{Tape}(c, s, h)$ where $s \neq$ transition_state or $h=$ false;

- otherwise, then Output $\left(p_{i-1}\right)=\operatorname{Error}^{4}$ (current_state, tape_content, $\left.j-1\right)$.

10. If Output $\left(p_{i}\right)=\operatorname{Error}^{5}(x)$

- if Output $\left(p_{i-1}\right) \neq \operatorname{Error}^{5}$ then $\operatorname{Input}\left(p_{i}\right)=\operatorname{Tape}(c, s, h)$ where $h=$ true and $x=0$.

11. If Output $\left(p_{i}\right)=$ Error then one of the following condition holds:

- $\operatorname{Input}\left(p_{i}\right) \neq \operatorname{Start}(\phi)$ and $p_{i}$ has no predecessors;

- Input $\left(p_{i}\right)=\operatorname{Start}(\phi)$ and $p_{i}$ has a predecessor;

- Input $\left(p_{i-1}\right)$ or Output $\left(p_{i-1}\right)$ is Empty;

- Output $\left(p_{i-1}\right)=$ Error;

- Output $\left(p_{i-1}\right)=\operatorname{Error}^{0}(j), j>0$, and

- if $j=1$ then $\operatorname{Input}\left(p_{i-1}\right) \neq$ Separator;

- if $j \geq 2$ then either $\operatorname{Input}\left(p_{i-1}\right) \neq$ Tape, or $\operatorname{Input}\left(p_{i-1}\right)=\operatorname{Tape}(c, s, h)$ and:

$*$ if $j=2$ either $c \neq L$, or $s \neq q_{0}$ or $h=$ false;

$*$ if $2<j \leq B$ either $c \neq 0$, or $s \neq q_{0}$ or $h=$ true; 
* if $j=B+1$, either $c \neq R$, or $s \neq q_{0}$ or $h=$ true;

- Input $\left(p_{i}\right)=$ Separator and Output $\left(p_{i-1}\right)=\operatorname{Error}^{1}(x)$ where $x \neq B$;

- Input $\left(p_{i}\right) \neq$ Separator and Output $\left(p_{i-1}\right)=\operatorname{Error}^{1}(B)$;

- Output $\left(p_{i-1}\right)=\operatorname{Error}^{2}(x, j)$ where $j=B+1$;

- Output $\left(p_{i-1}\right)=$ Error $^{3}$;

- $p_{i-1}$ is an "Error ${ }^{4}$ final node";

- Output $\left(p_{i-1}\right)=\operatorname{Error}^{5}(x)$ and $\operatorname{Input}\left(p_{i-1}\right)=\operatorname{Tape}(c, s, h)$ where $h=$ true and $x=1$.

12. If Output $\left(p_{i}\right)$ is of type $\operatorname{Error}^{x}$, then Output $\left(p_{i-1}\right)$ must not be of type $\operatorname{Error}^{y}$ where $y \neq x$.

The following property directly holds by definition of the constraints.

Property 1. Each node is able to locally check all constraints by just inspecting its own input and output, and the ones of its predecessor (if it exists).

\subsection{Upper Bound on the Complexity of the $\mathrm{LCL}$}

We need to consider two possible scenarios: either $M_{B}$ terminates within time $T$, or $M_{B}$ loops. In the case in which $M_{B}$ loops, we show a simple $O(n)$ algorithm that solves the LCL $\Pi_{M_{B}}$. As we know, any problem for which a solution exists can be solved in $O(n)$ rounds in the LOCAL model by gathering all the graph and solving the problem locally. There always exists a solution for problem $\Pi_{M_{B}}$ if $M_{B}$ loops, in fact:

- If $\operatorname{Input}\left(p_{0}\right)=\operatorname{Start}(\phi)$, then all nodes output $\phi$, even if there are errors in the machine encoding.

- Otherwise, if Input $\left(p_{0}\right) \neq \operatorname{Start}(\phi)$, all nodes output Error.

It is easy to see that this output satisfies the LCL constraints described above.

Suppose that $M_{B}$ terminates. In this case, we show how to solve the $\mathrm{LCL}$ problem $\Pi_{M_{B}}$ in constant time. More precisely, if $M_{B}$ terminates in $T$ rounds, we show a distributed algorithm that solves $\Pi_{M_{B}}$ in $T^{\prime}=2+(B+1) T$ rounds. Each node $v$ starts by gathering its $T^{\prime}$-radius neighborhood $B_{v}\left(T^{\prime}\right)$. Notice that, by definition, if the input is a good input, then for each node $v$ that is taking part in the encoding of the execution of $M_{B}$ (i.e., Input $(v) \neq$ Empty), $B_{v}\left(T^{\prime}\right)$ contains $p_{0}$. Hence, if a node $v$ does not see $p_{0}$ after gathering its ball $B_{v}\left(T^{\prime}\right)$, it means that the input is not a good input. So, after gathering its $T^{\prime}$-radius ball, each node $v$ does the following.

- If Input $(v)=$ Empty, then Output $(v)=$ Empty.

- If $B_{v}\left(T^{\prime}\right)$ does not contain $p_{0}$, or if $\operatorname{Input}\left(p_{0}\right) \neq \operatorname{Start}(\phi)$, then $v$ outputs Error.

- If $B_{v}\left(T^{\prime}\right)$ is a good input, then $v$ outputs $\operatorname{Start}(\phi)$.

The remaining case that we still need to handle is when $B_{v}\left(T^{\prime}\right)$ contains $p_{0}, \ln \operatorname{lnt}\left(p_{0}\right)=\operatorname{Start}(\phi)$, but $B_{v}\left(T^{\prime}\right)$ does not look like a good input. We want nodes to produce a proof of an error in some consistent way. Thus, we show that nodes can identify the first error and produce a proof based on that. First of all, notice that, since $v$ sees the first node in the path, $v$ can compute its position $i$ on the path. Also, node $v$ can identify who is the first node $u$ not satisfying the constraints of being a good input. Let $j$ be the position of $u$ in the path, that is $u=p_{j}$. Now we distinguish the following cases based on $B_{u}(B+2)$ (the output of each node will be determined by the first case encountered in the following list). 
1. If Input $\left(p_{j}\right)=\operatorname{Start}(\phi)$ and $j \neq 0$, then, if $i<j$, Output $(v)=\operatorname{Start}(\phi)$; otherwise Output $(v)=$ Error.

2. If $j \leq B+1$, it means that either the initial state is encoded incorrectly, or the tape is not initialized correctly, or the head is not initialized on the correct position. In this case, if $i \leq j$, then Output $(v)=\operatorname{Error}^{0}(i)$, otherwise Output $(v)=$ Error.

3. If Input $\left(p_{j-(B+1)}\right)=$ Separator and $\operatorname{Input}\left(p_{j}\right) \neq$ Separator, then the length of the tape is too long, and $u$ expected to have in input Separator. Then, if $i<j-(B+1)$, Output $(v)=\operatorname{Start}(\phi)$; if $i>j$ then Output $(v)=$ Error; otherwise, Output $(v)=\operatorname{Error}^{1}(i-j+B+1)$.

4. If $\operatorname{Input}\left(p_{j}\right)=$ Separator and there exists a $k$ such that $1 \leq j-k<B+1$ such that $\operatorname{Input}\left(p_{k}\right)=$ Separator, then the length of the tape is too short, and $u$ did not expect to have a separator. In this case, if $i<k$ then Output $(v)=\operatorname{Start}(\phi)$; if $i \geq j$ then Output $(v)=$ Error; otherwise Output $(v)=\operatorname{Error}^{1}(k-i)$.

5. If Input $\left(p_{j-(B+1)}\right)=\operatorname{Tape}(c, s, h)$ where $h=$ false, $c=x$, and $\operatorname{Input}\left(p_{j}\right)=\operatorname{Tape}\left(c^{\prime}, s^{\prime}, h^{\prime}\right)$, where $c^{\prime} \neq x$, then the tape of $M_{B}$ has been copied incorrectly. In this case, if $i<j-(B+1)$, then Output $(v)=\operatorname{Start}(\phi)$; if $i>j$ then Output $(v)=\operatorname{Error}$; otherwise, Output $(v)=\operatorname{Error}^{2}(x, i-j+$ $B+1)$.

6. If Input $\left(p_{j}\right)=\operatorname{Tape}(c, s, h)$ and $\operatorname{Input}\left(p_{j-1}\right)=$ Separator and there exists a $k<j+B$ such that $\operatorname{Input}\left(p_{k}\right)=\operatorname{Tape}\left(c^{\prime}, s^{\prime}, h^{\prime}\right)$ and that $s \neq s^{\prime}$, it means that nodes have inconsistent states. Consider the minimum $k$ satisfying the constraints. If $i<k$ then Output $(v)=\operatorname{Start}(\phi)$; if $i>k$ then Output $(v)=$ Error; otherwise, Output $(v)=$ Error $^{3}$.

7. If none of the above is satisfied, it means that there exist a $k$ satisfying $j-k \leq B+2$, such that $\operatorname{Input}\left(p_{k}\right)=\operatorname{Tape}(c, s, h)$ and $h=$ true. Let (transition_state, new_content, $\left.\epsilon\right)=\delta(s, c)$. It holds that if $\epsilon$ is $\leftarrow$, - , or $\rightarrow$, then $j-k$ is respectively $B, B+1$, or $B+2$. If Input $\left(p_{j}\right)=\operatorname{Tape}\left(c^{\prime}, s^{\prime}, h^{\prime}\right)$, where either $h^{\prime}=$ false, or transition_state $\neq s^{\prime}$, or $s$ is a final state, then there is some error in the transition (this captures also the case where there is no head). If $i<k$, then $\operatorname{Output}(v)=\operatorname{Start}(\phi)$; if $i>j$ then Output $(v)=\operatorname{Error}$; otherwise, Output $(v)=\operatorname{Error}^{4}(s, c, k-i)$. Notice that this case captures also the one where the head is missing.

8. If Input $\left(p_{j}\right)=\operatorname{Tape}(c, s, h)$ where $h=$ true, since all the above cases are not satisfied, it means that there exists a $k$, such that $\operatorname{Input}\left(p_{k}\right)=\operatorname{Tape}\left(c^{\prime}, s^{\prime}, h^{\prime}\right), h^{\prime}=$ true, $|j-k|<B$ and all nodes $p_{\min (j, k)}, \ldots, p_{\max (j, k)}$ are labeled with some Tape. That is, there are at least two heads, one on node $p_{j}$ and one on node $p_{k}$. In this case, if $i<\min (j, k)$, then Output $(v)=\operatorname{Start}(\phi)$; if $i>\max (j, k)$ then $\operatorname{Output}(v)=\operatorname{Error}$; if $i=\min (j, k)$ then Output $(v)=\operatorname{Error}^{5}(0)$, otherwise Output $(v)=\operatorname{Error}^{5}(1)$.

If the path encodes a good input, every node taking part in the encoding of the execution of $M_{B}$ outputs $\operatorname{Start}(\phi)$, and in this case it is easy to see that the output satisfies the LCL constraints.

Therefore, assume that the path does not correctly encode the execution of $M_{B}$ starting from the correct tape content. First of all, notice that the algorithm handles all possible errors in the machine encoding, that is, if the input is not good, at least one case of the list applies. Consider all nodes $v$ that do not have in input Empty, that is, all nodes taking part in the encoding of the execution of $M_{B}$. If node $v$ sees the first node $p_{0}$, i.e., if the distance between $p_{0}$ and $v$ is at most $T^{\prime}$ (notice that a good input has length $T^{\prime}$ ), then it is easy to see that the output satisfies the LCL constraints. Some care is needed in the case where a node $v$ outputs a generic error Error and $v$ does not see $p_{0}$ : we need to show that also in this case the output is valid, meaning that the $\mathrm{LCL}$ 
constraints are satisfied. In this case, the distance between $p_{0}$ and $v$ is strictly greater than $T^{\prime}$, and since the encoding of the execution of $M_{B}$ is not correct, then

- either the path $\left(p_{0}, \ldots, v\right)$ does not correctly encode the execution of $M_{B}$,

- or $M_{B}$ is not correctly initialized and it loops.

In the first case, some node on the path between $p_{0}$ and $v$ will output some specific error Error ${ }^{x}$ where $x>0$, while in the second case initial nodes will output Error ${ }^{0}$. In both scenarios the constraints for Error are satisfied. The complexity of the algorithm is $O(B \cdot T)$.

\subsection{Lower Bound on the Complexity of the $\mathrm{LCL}$}

Let us define $T^{\prime \prime}$ as follows. If $M_{B}$ terminates in time $T$, then $T^{\prime \prime}=T^{\prime}=2+(B+1) T$. If $M_{B}$ loops, then $T^{\prime \prime}=n$. We prove a lower bound on the complexity of $\Pi_{M_{B}}$ of $\Omega\left(T^{\prime \prime}\right)$ rounds, by showing that $\Omega\left(T^{\prime \prime}\right)$ rounds are needed in the case where the input is a good input. In particular, we show that, in a good input, for all nodes $v$ such that $\operatorname{Input}(v) \neq \operatorname{Empty}$, Output $(v)$ must be $\operatorname{Start}(\phi)$. The result then comes from the fact that, for some nodes, it requires $\Omega\left(T^{\prime \prime}\right)$ rounds in order to see if $\phi=a$ or $\phi=b$.

First of all, we ignore nodes that have in input Empty since, in a good input, they are at distance at least $T^{\prime \prime}+1$ from $p_{0}$, the first node of the path. Hence, assume that a node $v$ not having Empty in input does not output Start $(\phi)$. In this case, $v$ can either output a generic error Error, or a specific error Error ${ }^{x}$. If all nodes output Error, the verifier rejects on $p_{0}$. If all nodes, starting from a node $p_{i}$ where $i>0$, output Error, and all nodes $p_{i^{\prime}}$ with $i^{\prime}<i$ output Start $(\phi)$, then the verifier rejects on $p_{i}$. Therefore, let us assume that there is at least a node that outputs a specific error Error ${ }^{x}$. We write $\operatorname{succ}(v)$ and $\operatorname{dist}(u, v)$ to denote respectively the successor of a node $v$ in the path, and the distance between two nodes $u$ and $v$ in the path.

- If $x=0$, the verifier accepts only if this error produces a chain that starts from $p_{0}$ and proceeds with increasing values. In order to be accepted, this chain must end at a node $w^{\prime}$, and $\operatorname{succ}\left(w^{\prime}\right)$ must output Error. Then, $\operatorname{succ}\left(w^{\prime}\right)$ must witness that $w^{\prime}$ indeed has a local error in the machine initialization, which is not possible in a good input.

- If $x=1$, we could have two cases:

- there is a chain of increasing values that starts from a node $w$ with Input $(w)=$ Separator, and ends on a node $w^{\prime}$ such that $\operatorname{dist}\left(w, w^{\prime}\right)<B$, Input $\left(\operatorname{succ}\left(w^{\prime}\right)\right)=$ Separator, and Output $\left(\operatorname{succ}\left(w^{\prime}\right)\right)=$ Error (the tape is too short);

- there is a chain of increasing values that starts from a node $w$ with Input $(w)=$ Separator, and ends on a node $w^{\prime} \operatorname{such}$ that $\operatorname{dist}\left(w, w^{\prime}\right)=B, \operatorname{Input}\left(\operatorname{succ}\left(w^{\prime}\right)\right) \neq$ Separator, and Output $\left(\operatorname{succ}\left(w^{\prime}\right)\right)=$ Error (the tape is too long).

Since, in a good input, the distance between two nodes having in input Separator is always $B+1$, the above scenarios are not possible.

- If $x=2$, there must be a chain of length exactly $B+1$, starting from a node $w$ having Input $(w)=\operatorname{Tape}(c, s, h)$, where $c=x \in\{0,1\}, h=$ false, and ending on a node $w^{\prime}$ such that $\operatorname{dist}\left(w, w^{\prime}\right)=B+1$, and Input $\left(w^{\prime}\right)=\operatorname{Tape}\left(c^{\prime}, s^{\prime}, h^{\prime}\right)$, where $c^{\prime} \neq x$. In a good input, the tape content of nodes $w$ and $w^{\prime}$ must be the same.

- If $x=3$, it means that there must exist two neighbors having two different states, and this can not happen in a good input. 
- If $x=4$, there must be a chain that propagates the old state and old input, and the verifier accepts only if acknowledges that the transition has been wrongly encoded, which can not the case in a good input.

- If $x=5$, there must be a chain of length at least 2 not passing through nodes having in input Separator, starting from a node $w$ with $\operatorname{Input}(w)=\operatorname{Tape}(c, s, h)$ where $h=$ true, and ending on a node $w^{\prime}$ with $\operatorname{Input}\left(w^{\prime}\right)=\operatorname{Tape}\left(c^{\prime}, s^{\prime}, h^{\prime}\right)$ where $h^{\prime}=$ true. This is not possible on a good input.

Therefore, since nodes can not output any kind of error, and since Empty is not a valid output for the nodes encoding the LBA, then these nodes must output Start $(\phi)$, where the value of $\phi$ matches the input of the first node of the path. Hence, $\Pi_{M_{B}}$ requires $\Omega\left(T^{\prime \prime}\right)$.

\subsection{Normalizing an LCL Problem}

We now show how to $\beta$-normalize an $\mathrm{LCL} \Pi_{M_{B}}$ and obtain a new LCL having roughly the same time complexity. We define three different verifiers depending on their view.

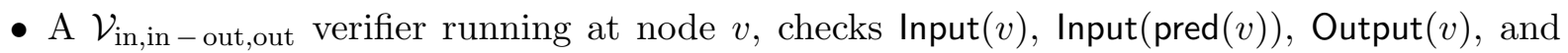
Output $((\operatorname{pred}(v))$.

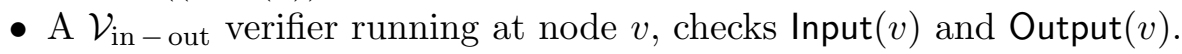

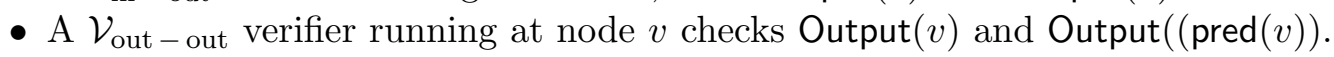

Lemma 2. Consider an $\mathrm{LCL} \mathcal{P}$ with $\left|\Sigma_{\text {in }}^{\mathcal{P}}\right|=\alpha$ and $\left|\Sigma_{\text {out }}^{\mathcal{P}}\right|=\beta$ that can be solved in time $T$ and can be locally checked with a $\mathcal{V}_{\mathrm{in}, \mathrm{in}-\mathrm{out}, \mathrm{out}}$ verifier. It is possible to define an $\mathrm{LCL} \mathcal{P}^{\prime}$ such that $\left|\Sigma_{\mathrm{in}}^{\mathcal{P}^{\prime}}\right|=\alpha$ and $\left|\Sigma_{\text {out }}^{\mathcal{P}^{\prime}}\right|=\alpha \cdot \beta$ that can be solved in time $T$ and can be locally checked with a $\mathcal{V}_{\text {in }- \text { out }}$ and $a$ $\mathcal{V}_{\text {out - out verifier. }}$

Proof. We define $\Sigma_{\text {in }}^{\mathcal{P}^{\prime}}=\Sigma_{\text {in }}^{\mathcal{P}}$, and $\Sigma_{\text {out }}^{\mathcal{P}^{\prime}}=\Sigma_{\text {out }}^{\mathcal{P}} \times \Sigma_{\text {out }}^{\mathcal{P}}$. Let Output $(v)=$ (in, out) $\in \Sigma_{\text {out. }}^{\mathcal{P}^{\prime}}$. Let Output $(\operatorname{pred}(v))=\left(\right.$ in $^{\prime}$, out $\left.^{\prime}\right) \in \Sigma_{\text {out }}^{\mathcal{P}^{\prime}}$. The $\mathcal{V}_{\text {in }- \text { out }}$ verifier checks that Input $(v)=$ in. The $\mathcal{V}_{\text {out }- \text { out }}$

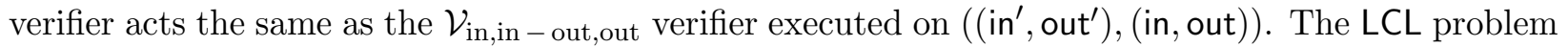
$\mathcal{P}^{\prime}$ can be solved with the following algorithm at each node $v$.

- Gather the ball $B_{v}(T)$.

- Simulate the original algorithm on $B_{v}(T)$; let out be the output of this simulation.

- Output $(\operatorname{Input}(v)$, out$)$.

It is easy to check that this output is valid for the problem $\mathcal{P}^{\prime}$, and that it requires $T$ rounds. Also, note that it is not possible to solve $\mathcal{P}^{\prime}$ faster than $T$. In fact, in order to satisfy the $\mathcal{V}_{\text {in }- \text { out verifier, }}$

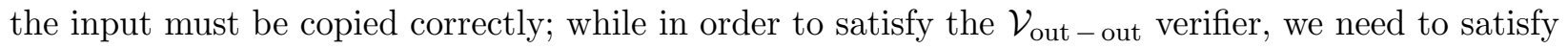

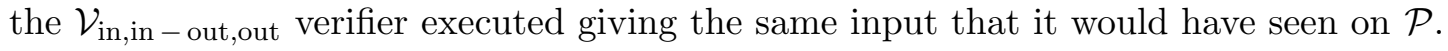

Lemma 3. Consider an $\mathrm{LCL} \mathcal{P}$ with $\left|\Sigma_{\mathrm{in}}^{\mathcal{P}}\right|=\alpha$ and $\left|\Sigma_{\text {out }}^{\mathcal{P}}\right|=\beta$ that can be solved in time $T$ and can be locally checked with a $\mathcal{V}_{\text {in -out }}$ and a $\mathcal{V}_{\text {out-out }}$ verifier. We can define a $\beta^{\prime}$-normalized $\mathrm{LCL} \mathcal{P}^{\prime}$ with $\beta^{\prime}=\left|\Sigma_{\text {out }}^{\mathcal{P}^{\prime}}\right|=2^{\gamma} \cdot\left(\left|\Sigma_{\text {out }}^{\mathcal{P}}\right|+3\right)$ that can be solved in time $\Theta(\gamma \cdot T(n / \gamma))$, where $\gamma=2\lceil\log \alpha\rceil+3$.

Proof. In the following we will exploit the ability of an algorithm to work on identifiers that can be polynomial in the size of the graph. In particular, we assume that if an algorithm works on an instance with IDs in the range $1, \ldots, r$, then it works also on an instance with IDs in the range $1, \ldots, \gamma \cdot r$. Then, we show how to define an $\operatorname{LCL} \mathcal{P}^{\prime}$ such that:

- if the input instance encodes a virtual instance for the problem $\mathcal{P}$, it is required to solve $\mathcal{P}$ on the virtual instance; 
- otherwise, it is required to prove that the encoding is wrong.

Let $\mathcal{V}_{\text {in - out }}^{\prime}$ and $\mathcal{V}_{\text {out - out }}^{\prime}$ be the verifiers of our $\beta^{\prime}$-normalized LCL.

Encoding $\mathcal{P}$ in $\mathcal{P}^{\prime}$. We start by defining how to encode an instance of $\mathcal{P}$ of size $n$, as an instance of $\mathcal{P}^{\prime}$ of size $N=\gamma \cdot n$. We denote with $p_{0}, \ldots, p_{n-1}$ and $p_{0}^{\prime}, \ldots, p_{\gamma \cdot n-1}^{\prime}$ respectively the instance of $\mathcal{P}$ and the one of $\mathcal{P}^{\prime}$. Let $a=\lceil\log \alpha\rceil$. For the sake of simplicity, let us rename nodes $p_{\gamma i}^{\prime}, \ldots, p_{\gamma(i+1)-1}^{\prime}$, where $0 \leq i \leq n-1$, as $q_{0}^{i}, \ldots, q_{2 a+2}^{i}$ (notice that $2 a+2=\gamma-1$ ). The first $a+1$ nodes, $q_{0}^{i}, \ldots, q_{a}^{i}$, have input 1 , while nodes $q_{a+1}^{i}$ and $q_{2 a+2}^{i}$ have input 0 . Each of the remaining $a$ nodes, $q_{a+2}^{i}, \ldots, q_{2 a+1}^{i}$, has in input one bit of the binary representation of $\operatorname{Input}\left(p_{i}\right)$, in some fixed order (see Figure 3 for an illustration).

The $\mathcal{V}_{\text {in - out }}^{\prime}$ Verifier. The set of output labels of $\mathcal{P}^{\prime}$ is $\Sigma_{\text {out }}^{\mathcal{P}^{\prime}}=2^{\gamma} \times\left(\Sigma_{\text {out }} \cup\left\{E_{r}, E, E_{l}\right\}\right)$. Let $q_{j}^{i}$ be a node of the instance of $\mathcal{P}^{\prime}$ where $0 \leq i \leq n-1$ and $0 \leq j \leq 2 a+2$. Let $\operatorname{Input}\left(q_{j}^{i}\right) \in\{0,1\}$, and let Output $\left(q_{j}^{i}\right)=\left(\left(b_{0}, \ldots, b_{2 a+2}\right)\right.$, out $) \in \Sigma_{\text {out }}^{\mathcal{P}^{\prime}}$. The $\mathcal{V}_{\text {in }- \text { out }}^{\prime}$ verifier running at $q_{j}^{i}$ checks that

- Input $\left(q_{j}^{i}\right)=b_{0}$, and

- if out $\in \Sigma_{\text {out }}^{\mathcal{P}}$, then

- if all bits in $b_{0}, \ldots, b_{a}$ are $1 \mathrm{~s}$, checks that the original $\mathcal{V}_{\text {in - out }}$ verifier accepts on ( $x$, out), where $x$ is obtained by recovering the input $p_{i}$ for the original algorithm from $b_{a+2}, \ldots, b_{2 a+1}$.

The $\mathcal{V}_{\text {out - out }}^{\prime}$ Verifier. Let the output of $p_{j}^{i}$ be $\left(\left(b_{0}, \ldots, b_{2 a+2}\right)\right.$, out $) \in \Sigma_{\text {out }}^{\mathcal{P}^{\prime}}$ and the output of the predecessor of $p_{j}^{i}$ be $\left(\left(b_{0}^{\prime}, \ldots, b_{2 a+2}^{\prime}\right)\right.$, out $\left.{ }^{\prime}\right) \in \Sigma_{\text {out }}^{\mathcal{P}^{\prime}}$ The O-O verifier first checks that

- $b_{0}=b_{1}^{\prime}, b_{1}=b_{2}^{\prime}, \ldots, b_{2 a+1}=b_{2 a+2}^{\prime}$, and

- if out $\in \Sigma_{\text {out }}^{\mathcal{P}}$ and out ${ }^{\prime} \in \Sigma_{\text {out }}^{\mathcal{P}}$, then

- if at least one bit in $b_{0}, \ldots, b_{a}$ is 0 , then out $=$ out $^{\prime}$,

- if all bits in $b_{0}, \ldots, b_{a}$ are $1 \mathrm{~s}$, then check that the original $\mathcal{V}_{\text {out - out }}$ executed on (out ${ }^{\prime}$, out) accepts.

Dealing with Errors. We now add some constraints to handle the case in which out $\notin$ $\Sigma_{\text {out }}^{\mathcal{P}}$. Let the output of $p_{j}^{i}$ be $\left(\left(b_{0}, \ldots, b_{2 a+2}\right)\right.$, out $)$ and the output of the predecessor of $p_{j}^{i}$ be $\left(\left(b_{0}^{\prime}, \ldots, b_{2 a+2}^{\prime}\right)\right.$, out $\left.^{\prime}\right)$. The $\mathcal{V}_{\text {in - out }}^{\prime}$ verifier additionally checks that, if out $=E$, then the encoding is not locally valid, that is,

- either

- there are two numbers $x, y \geq 0, x+y \leq a$, such that $b_{0}, \ldots, b_{x-1}$ and $b_{2 a+3-y}, \ldots, b_{2 a+2}$ are all equal to $1, b_{x}=0, b_{2 a+2-y}=0$, and

- there is not a contiguous sequence of length $a+1$ of all $1 \mathrm{~s}$ in $b_{0}, \ldots, b_{2 a+2}$,

- or $b_{0}, \ldots, b_{a}$ are all 1 s but either $b_{a+1} \neq 0$ or $b_{2 a+2} \neq 0$.

The $\mathcal{V}_{\text {out - out }}^{\prime}$ verifier running on $p_{j}^{i}$ additionally checks that,

- if out $=E_{l}$, then $p_{j}^{i}$ must have a predecessor, and it must hold that out ${ }^{\prime} \notin\left\{E_{r}\right\} \cup \Sigma_{\text {out }}^{\mathcal{P}}$;

- if out $\in \Sigma_{\text {out }}^{\mathcal{P}}$, if $p_{j}^{i}$ has a predecessor, then out' must be different from $E_{r}$;

- if out $=E_{r}$, then $p_{j}^{i}$ must have a successor. 
Let $N$ be the size of the graph. An algorithm solving $\mathcal{P}^{\prime}$ in $(\gamma+1) \cdot T$ rounds does the following at each node $v^{\prime}$ :

- Gather the ball $B_{v^{\prime}}((\gamma+1) \cdot T)$.

- If $B_{v^{\prime}}((\gamma+1) \cdot T)$ looks like a correct encoding of an input instance of $\mathcal{P}$

- let $u^{\prime}$ be the nearest left node having input 1 and other $a$ successors, succ ${ }^{(1)} u^{\prime}, \ldots, \operatorname{succ}^{(a)} u^{\prime}$, having also input 1

- Compute the virtual instance for $\mathcal{P}$ (setting the IDs to be the same of the nodes satisfying the above)

- Simulate the original algorithm on the virtual instance by setting $n=N / \gamma$, let out be the output of $u^{\prime}$

- Output $\left(\left(\operatorname{Input}\left(v^{\prime}\right), \operatorname{Input}\left(\operatorname{succ}^{(1)} v^{\prime}\right), \ldots, \operatorname{Input}\left(\operatorname{succ}^{(\gamma-1)} v^{\prime}\right)\right.\right.$, out $)$

- Otherwise,

- if there is a local error, output $E$

- if the nearest error is on the left, output $E_{l}$

- otherwise, output $E_{r}$

It is easy to check that the output of the algorithm satisfies the constraints. In order to show a lower bound for the new LCL, we now show that it is not possible to produce errors in a graph that is a valid encoding. In fact, nodes can not cheat by wrongly outputting the input of the neighbors, otherwise either the input-output verifier notices inconsistencies on the first bit, or the output-output verifier notices inconsistencies on the other bits. Then, on a valid encoding, no input satisfies the constraints that allows to produce $E$ as output. Finally, the constraints impose that a chain of $E_{r}$ or $E_{l}$ points to a node that is outputting $E$.

Note that, if the original $\mathrm{LCL}$ has complexity $T$, then the new $\mathrm{LCL}$, on instances of size $N=\gamma \cdot n$, has complexity $\Theta(\gamma(T(n)))=\Theta\left(\gamma \cdot T\left(\frac{N}{\gamma}\right)\right)$.

\subsection{Hardness Results}

Theorem 4. There are $\beta$-normalized $\mathrm{LCL}$ s that can be solved in constant time but the distributed time complexity is $2^{\Omega(\beta)}$.

Proof. The complexity of $\Pi_{M_{B}}$ is $\Theta(B \cdot T)$ if $M_{B}$ terminates in $T$ steps. $\left|\Sigma_{\text {in }}\right|=O(1)$ and $\left|\Sigma_{\text {out }}\right|=\Theta(B)$. We can convert it to an $L C L$ where $\left|\Sigma_{\text {in }}\right|=2,\left|\Sigma_{\text {out }}\right|=\Theta(B)$, and the complexity is still $\Theta(B \cdot T)$. There exist LBAs that terminate in $2^{\Theta(B)}$ steps (e.g. a binary counter). Thus, the complexity of the obtained $\mathrm{LCL}$ is $\Theta\left(B \cdot 2^{\Theta(B)}\right)$, that is $2^{\Omega(B)}=2^{\Omega\left(\left|\Sigma_{\text {out }}\right|\right)}=2^{\Omega(\beta)}$.

Theorem 5. It is PSPACE-hard to distinguish whether a given $\mathrm{LCL}$ problem $\mathcal{P}$ with input labels can be solved in $O(1)$ time or needs $\Omega(n)$ time on globally oriented path graphs.

Proof. It is PSPACE-hard to distinguish whether a given LBA terminates or loops (see e.g. [10]). Note that the description of a $\beta$-normalized LCL has size $O\left(\beta^{2}\right)$. In order to decide if a $\beta$-normalized version of a problem in $\Pi$ requires $O(1)$ or $\Omega(n)$ we need to decide if its associated LBA, running on a tape of size $B=\Theta(\beta)$, terminates or loops, and this implies the theorem. 


\subsection{Extending the Results to Undirected Cycles}

We show how to extend the above results, which apply to globally oriented paths, to the case where the input graph is an undirected path or an undirected cycle. We first focus on showing how to adapt these results to undirected paths. Given a $\beta$-normalized LCL $\mathcal{P}$ defined on directed paths, we can define an $\operatorname{LCL} \mathcal{P}^{\prime}$ in which the set of input labels is $\Sigma_{\text {in }}^{\prime}=\{0,1\} \times\{0,1,2\}$, and the set of output labels is $\Sigma_{\text {out }}^{\prime}=\{0,1,2\} \times\{1, \ldots, \beta, E\}$. Let $\mathcal{V}_{\text {in }- \text { out }}$ and $\mathcal{V}_{\text {out - out }}$ be the verifiers of the $\beta$-normalized LCL $\mathcal{P}$, and let $\mathcal{V}_{\text {in - out }}^{\prime}$ and $\mathcal{V}_{\text {out - out }}^{\prime}$ be the verifiers of the $\operatorname{LCL} \mathcal{P}^{\prime}$. The idea is that we can use 3 symbols to give an orientation as input to the nodes, by giving 0 to the first node, 1 to the second, 2 to the third, 0 to the fourth, and so on. Nodes must copy their orientation number to the output, and then, if the given orientation is consistent, nodes are required to solve the original problem $\mathcal{P}$. On the other hand, if the orientation is not consistent, nodes are allowed to output an error $E$. Also, in order to avoid the need of error pointers, we allow nodes to treat the places where the orientation is not consistent, as a place where the path ends.

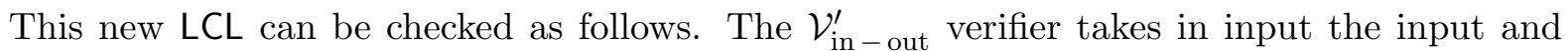
the output of the current node (as before) and first checks that the orientation has been copied

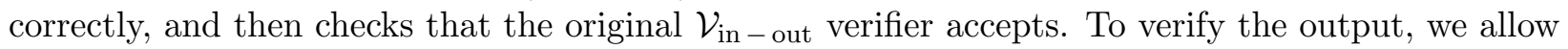
the $\mathcal{V}_{\text {out - out }}^{\prime}$ verifier to see slightly more than the original verifier $\mathcal{V}_{\text {out - out }}$. The $\mathcal{V}_{\text {out - out verifier }}^{\prime}$ sees a triple containing the output of the node and the outputs of its neighbors. Note that the verifier does not know the orientation of the path (and the orientation of the triple), but it can recover it from the output of the nodes (that contains a copy of the orientation given as input). Then the $\mathcal{V}_{\text {out - out }}^{\prime}$ verifier checks that, if the node outputted $E$, the orientation is indeed wrong. If

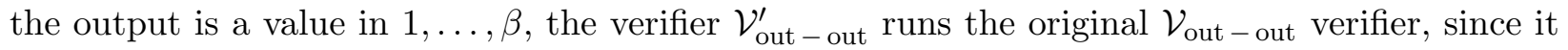
can compute which neighbor is the predecessor. It is easy to see that the complexity of $\mathcal{P}^{\prime}$ is the same as the one of $\mathcal{P}$.

The LCL description of $\mathcal{P}^{\prime}$, that is, the size of its input, its output, and its verifier, is now $O\left(\beta^{3}\right)$, therefore the hardness result still applies.

We now show how an LCL for paths can be converted to an LCL for cycles. The idea is the following. On a cycle, we give an additional input to each node in $\{0,1\}$. Nodes marked as 1 are exempt to solve the problem and act as separators between the other nodes. That is, nodes are required to solve the original problem on the subpaths that lie between nodes marked as 1 . It may be the case that no node has 1 as input. In this case we allow nodes to output a special error. If a node decides to output this error, both its neighbors must output the same, that is, all nodes must output the same. We impose the constraint that a node marked as 1 can not output this error. A worst case instance would be the one in which one node is marked 1 and all other nodes are marked 0 - this would represent a path with a length that is roughly equal to the one of the cycle. There is one case that requires a bit of care: if all nodes are marked 0, but the original problem can be solved in sublinear time, nodes could not be able to coordinate to produce the special error. For our purpose, it is possible to check that, if we consider the problem $\Pi_{M_{B}}$ previously defined in the case in which $M_{B}$ terminates, in an instance in which nobody has a predecessor, nodes can efficiently solve the problem by just outputting Error or Empty, depending on their input.

\subsection{Encoding Input Labels as Trees}

In this section we demonstrate a reduction from the $\mathrm{LCL}$ problem $\mathcal{P}$ with input labels on any graph $G$ to an LCL problem $\mathcal{P}^{\star}$ without input labels on the modified graph $G^{\star}$. The modified graph $G^{\star}$ is the result of attaching a rooted tree to each $v \in V(G)$ that encodes the input label of $v$ for the $\mathrm{LCL}$ problem $\mathcal{P}$. The reduction allows us to extend the hardness proof to the case of LCL problems 
without input labels.

Encoding. Given a $2^{k}$-bit binary string $S=\left(s_{1}, \ldots, s_{2^{k}}\right)$, define $\operatorname{Enc}(S)$ as the rooted tree constructed as follows.

- Begin with the full binary tree which has $2^{k}$ leaves, and the distance from the root to each leaf is $k$.

- For each non-leaf node $v$, let $u$ be any one of its two children, and subdivide the edge $\{v, u\}$ into two edges $\{v, w\}$ and $\{w, u\}$, where $w$ is a new node. The node $w$ is designated as the left child of $v$.

- Let $U=\left(u_{1} \ldots, u_{2^{k}}\right)$ be the leaves ordered by the in-order traversal.

- For each $i \in\left[2^{k}\right]$, add two new nodes $x$ and $y$ as the children of $u_{i}$. If $s_{i}=1$, add two more new nodes $x^{\prime}$ and $y^{\prime}$ and the two edges $\left\{x, x^{\prime}\right\}$ and $\left\{y, y^{\prime}\right\}$.

The tree $\operatorname{Enc}(S)$ has maximum degree 3 , and all nodes are within distance $2(k+1)$ to the root. Given a graph $G$ such that each node $v \in V(G)$ is associated with an input label $L(v) \in \Sigma_{\text {in }}$, define $G^{\star}$ as the graph resulting from the following operations on $G$. For each node $v \in V(G)$, attach the rooted tree $T=\operatorname{Enc}(L(v))$ to $v$ by adding the edge $\{v, z\}$, where $z$ is the root of $T$. Notice that $\Delta\left(G^{\star}\right)=\max \{3, \Delta(G)+1\}$.

Decoding. Given a rooted tree $T=\operatorname{Enc}(S)$ for some $S \in\{0,1\}^{2^{k}}$, define $\operatorname{Dec}(T)=S$. The decoding can be done by the following procedure. Consider an in-order traversal of the tree such that (i) for each node $v$ such that exactly one of its children $w$ has degree 2, treat $w$ as the left child of $v$, (ii) print ' 1 ' if a node that has two children of degree 2 is encountered, (iii) print ' 0 ' if a node that has two children of degree 1 is encountered. Then the printed sequence is $S$.

The Modified LCL Problem $\mathcal{P}^{\star}$. Let $\mathcal{P}$ be an LCL problem with input labels. Suppose that the radius of $\mathcal{P}$ is $r$, and the maximum degree is $\Delta$. Set $k=\left\lceil\log \log \left|\Sigma_{\text {in }}\right|\right\rceil$, and let each label in $\Sigma_{\text {in }}$ be represented by a distinct $2^{k}$-bit string. The modified $L C L$ problem $\mathcal{P}^{\star}$, which does not require input label, is defined by the following rules. The set of the output labels of $\mathcal{P}^{\star}$ is $\Sigma_{\text {out }}$, the same as that of $\mathcal{P}$. Let $G^{\star}$ be a graph with maximum degree $\leq \Delta+1$.

- Define $G_{1}=G^{\star}$.

For each $2 \leq i \leq k+2$, define $G_{i}$ as the graph induced by nodes in $V\left(G^{\star}\right)-\bigcup_{j=1}^{i-1}\left(A_{j} \cup B_{j}\right)$.

For each $i \in[k+2]$, define $A_{i}=\left\{v \in V\left(G_{i}\right) \mid \operatorname{deg}_{G_{i}}(v)=1\right\}$.

For each $i \in[k+1]$, define $B_{i}=\left\{v \in V\left(G_{i}\right) \mid \operatorname{deg}_{G_{i}}(v)=2\right.$ and $\exists u \in A_{i}$ s.t. $\left.\{u, v\} \in E\left(G_{i}\right)\right\}$.

- Define $V_{\text {label }}=\bigcup_{j=1}^{k+2} A_{j} \cup \bigcup_{j=1}^{k+1} B_{j}$.

Define $V_{\text {main }}$ as the set of nodes in $V\left(G^{\star}\right) \backslash V_{\text {label }}$ that have exactly one neighbor in $V_{\text {label }}$.

- For each $v \in V_{\text {main }}$, define $L(v)$ as follows. Let $u$ be the unique node in $V_{\text {label }}$ adjacent to $v$, and let $T$ be the connected component induced by nodes in $V_{\text {label }}$ that contains $u$. Set $L(v)=\operatorname{Dec}(T)$ (with $u$ being the root of $T$ ). If the decoding procedure $\operatorname{Dec}(T)$ fails, simply set $L(v)$ as the first label in $\Sigma_{\text {in }}$.

- The output labeling, together with the input labeling defined by the function $L(\cdot)$, forms a legal labeling of the subgraph induced by the nodes in $V_{\text {main }}$ for $\mathcal{P}$. 
Notice that the connected components in $V_{\text {label }}$ are the trees encoding input labels, and the subgraph induced by the nodes in $V_{\text {main }}$ is $G$ (as long as $G$ does not contain isolated node). The function $L(v)$ recovers the input label of $v$ for each $v \in V(G)$. Given that $\mathcal{P}$ has a valid labeling on all graphs (resp., trees) of maximum degree $\Delta$, the modified $\mathrm{LCL}$ problem $\mathcal{P}^{\star}$ also has a valid labeling on all graphs (resp., trees) of maximum degree $\Delta+1$.

Reducing the Radius. The above definition of $\mathcal{P}^{\star}$ requires radius $r+O(k)$, as a node $v \in V_{\text {main }}$ needs $O(k)$ extra rounds to calculate $L(u)$ for all $u \in N^{r}(v) \cup V_{\text {main }}$. We present a simple modification that reduces the radius to only $r+O(1)$ at the cost of expanding the number of output labels from $\left|\Sigma_{\text {out }}\right|$ to $\left|\Sigma_{\text {out }}\right|+2^{2^{k}}<\left|\Sigma_{\text {out }}\right|+\left|\Sigma_{\text {in }}\right|^{2}$. The idea is to let nodes in $V_{\text {label }}$ to use output labels to pass the information stored at the leaves to the root based on local rules. Consider a connected component $T$ induced by nodes in $V_{\text {label }}$. The subgraph $T$ is interpreted as a tree rooted at the unique node in $T$ that is adjacent to some node in $V_{\text {main }}$.

Base Case: Let $v \in A_{2}$. If $v$ is adjacent to two nodes in $B_{1}$, the output label of $v$ is 1 ; otherwise the output label of $v$ is 0 .

Root / Degree-3 Nodes: Let $3 \leq i \leq k+2$, and let $v \in A_{i}$. Then $v$ has a unique neighbor $u_{\text {left }} \in B_{i+1}$ and a unique neighbor $u_{\text {right }} \in A_{i+1}$. Let $S_{\text {left }}$ be the output label of $u_{\text {left }}$, and let $S_{\text {right }}$ be the output label of $u_{\text {right }}$. Then the output label of $v$ is the binary string $S_{\text {left }} \circ S_{\text {right }}$.

Degree-2 Nodes: Let $3 \leq i \leq k+1$, and let $v \in B_{i}$. Then $v$ has a unique neighbor $u \in A_{i+1}$. The output label of $v$ is the same as the output label of $u$.

Thus, for each node $v \in V_{\text {main }}, L(v)$ is simply the output label of the unique node $u \in V_{\text {label }} \cap N(v)$.

Theorem 6. For any $\mathrm{LCL}$ problem $\mathcal{P}$ on any graph $G$ of maximum degree $\Delta$ that does not have isolated nodes, the following two statements are equivalent.

- The labeling $\mathcal{L}: V(G) \rightarrow \Sigma_{\text {out }}$ is a valid labeling of $G$ for the problem $\mathcal{P}$.

- There exists some labeling $\mathcal{L}^{\prime}$ of the nodes in $V_{\text {label }}$ such that $\mathcal{L}$ and $\mathcal{L}^{\prime}$ together form a valid labeling of $G^{\star}$ for the problem $\mathcal{P}^{\star}$.

Theorem 7. It is PSPACE-hard to distinguish whether a given $\mathrm{LCL}$ problem $\mathcal{P}$ without input labels can be solved in $O(1)$ time or needs $\Omega(n)$ time on trees with degree $\Delta=3$.

\section{Decidability}

In this section, we show that the two gaps $\omega(1)-o\left(\log ^{*} n\right)$ and $\omega\left(\log ^{*} n\right)-o(n)$ for LCL problems with input labels on paths and cycles are decidable. More specifically, given a specification of an $\mathrm{LCL}$ problem $\mathcal{P}$, there is an algorithm that outputs a description of an asymptotically optimal deterministic LOCAL algorithm for $\mathcal{P}$, as well as its time complexity.

We will prove the statements for the case of cycles, but the analogous results for cycles and paths follows as a simple corollary, as we can encode constraints related to degree- 1 nodes as constraints related to nodes adjacent to a special input label. Furthermore, having a promise that the input is a path does not change the time complexity of an $L C L$ problem: if a problem can be solved in time $T=o(n)$ in labeled paths, the same algorithm will solve it also in time $T=o(n)$ in labeled cycles.

The proof of Theorem 8 is in Section 4.2; the proof of Theorem 9 is in Section 4.5. 
Theorem 8. For any LCL problem $\mathcal{P}$ on cycle graphs, its deterministic LOCAL complexity is either $\Omega(n)$ or $O\left(\log ^{*} n\right)$. Moreover, there is an algorithm that decides whether $\mathcal{P}$ has complexity $\Omega(n)$ or $O\left(\log ^{*} n\right)$ on cycle graphs; for the case the complexity is $O\left(\log ^{*} n\right)$, the algorithm outputs a description of an $O\left(\log ^{*} n\right)$-round deterministic LOCAL algorithm that solves $\mathcal{P}$.

Theorem 9. For any LCL problem $\mathcal{P}$ on cycle graphs, its deterministic LOCAL complexity is either $\Omega\left(\log ^{*} n\right)$ or $O(1)$. Moreover, there is an algorithm that decides whether $\mathcal{P}$ has complexity $\Omega\left(\log ^{*} n\right)$ or $O(1)$ on cycle graphs; for the case the complexity is $O(1)$, the algorithm outputs a description of an $O(1)$-round deterministic LOCAL algorithm that solves $\mathcal{P}$.

Graph Notation. For convenience, in this section, a directed path $P$ with input labels is alternatively described as a string in $\Sigma_{\text {in }}^{k}$, where $k>0$ is the number of nodes in $P$. Similarly, an output labeling $\mathcal{L}$ of $P$ is alternatively described as a string in $\Sigma_{\text {out }}^{k}$. In subsequent discussion, we freely switch between the graph-theoretic notation and the string notation. Given an output labeling $\mathcal{L}$ of $P$, we say that $\mathcal{L}$ is locally consistent at $v$ if the input and output labeling assigned to $N^{r}(v)$ is acceptable for $v$. Note that $N^{r}(v)$ refers to the radius- $r$ neighborhood of $v$. Given two integers $a \leq b$, the notation $[a, b]$ represents the set of all integers $\{a, a+1, \ldots, b\}$. Given a string $w$, denote $w^{R}$ as the reverse of $w$.

Overview. Before we proceed, we briefly discuss the high level idea of the proofs. The main tool underlying the proofs is the "pumping lemma" which was developed in [6]. Intuitively, we classify the set of all input-labeled paths into a finite number of equivalence classes satisfying the following property. Let $P$ be a subpath of $G$, and let $P^{\prime}$ be another path that is of the same equivalence class as $P$. Given a complete legal labeling of $G$, if we let $G^{\prime}$ be the result of replacing $P$ with $P^{\prime}$, then it is always possible to extend this partial labeling of $G^{\prime}$ to a complete legal labeling by appropriately labeling $P^{\prime}$. The pumping lemma guarantees that for any path $P$ whose length is at least the pumping constant $\ell_{\text {pump }}$, and for any number $x \geq \ell_{\text {pump }}$, there is another path $P^{\prime}$ of length at least $x$ and $P^{\prime}$ is of the same equivalence class as $P$.

Informally, in the proof of Theorem 8, we show that any LCL problem $\mathcal{P}$ solvable in $o(n)$ rounds can be solved in $O\left(\log ^{*} n\right)$ rounds in the following canonical way based on a "feasible labeling function" $f$. Intuitively, a labeling function $f$ is feasible if for any given independent set $I$ that is sufficiently well-spaced, we can apply $f$ to assign the output labels to each $v \in I$ and its nearby neighbors locally such that this partial labeling can always be extended to a complete legal labeling. The $\omega\left(\log ^{*} n\right)-o(n)$ gap and the decidability result follows from these two claims.

- If there is an $o(n)$-round algorithm $\mathcal{A}$ that solves $\mathcal{P}$, then a feasible function $f$ exists. This is proved by first create an imaginary graph where some paths are extended using pumping lemmas, and then apply a simulation of $\mathcal{A}$ on the imaginary graph.

- Whether a feasible function exists is decidable. Intuitively, this is due to the fact that the number of equivalence classes is finite.

The proof of Theorem 9 is a little more complicated since the time budget is only $O(1)$, so we cannot even afford to find an MIS. To solve this issue, we decompose the cycle graph $G$ into paths with unrepetitive patterns and paths with repetitive patterns, in $O(1)$ rounds. For paths with unrepetitive patterns, we are able to compute a sufficiently well-spaced MIS in $O(1)$ rounds by making use of the irregularity of the input patterns. Paths with repetitive patterns are similar to the paths without input labels, and we will show that we can always label them by repetitive output patterns, given that the underlying $\mathrm{LCL}$ problem is $o\left(\log ^{*} n\right)$-time solvable. 

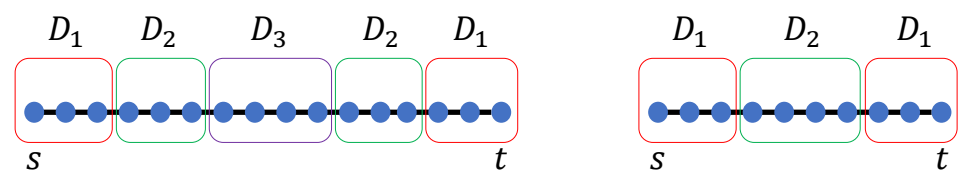

Figure 4: Illustration of the tripartition $\xi(P)=\left(D_{1}, D_{2}, D_{3}\right)$ with $r=3$.

\subsection{Pumping Lemmas for Paths}

Let $P=(s, \ldots, t)$ be a directed path, where each node has an input label from $\Sigma_{\text {in }}$. The tripartition of the nodes $\xi(P)=\left(D_{1}, D_{2}, D_{3}\right)$ is defined as follows:

$$
\begin{aligned}
& D_{1}=N^{r-1}(s) \cup N^{r-1}(t), \\
& D_{2}=\left(N^{2 r-1}(s) \cup N^{2 r-1}(t)\right) \backslash D_{1}, \\
& D_{3}=P \backslash\left(D_{1} \cup D_{2}\right) .
\end{aligned}
$$

See Figure 4 for an illustration. More specifically, suppose $P=\left(u_{1}, \ldots, u_{k}\right)$, and let $i \in[1, k]$. Then we have:

- $u_{i} \in D_{1}$ if and only if $i \in[1, r] \cup[k-r+1, k]$.

- $u_{i} \in D_{2}$ if and only if $i \in[r+1,2 r] \cup[k-2 r+1, k-r]$.

- $u_{i} \in D_{3}$ if and only if $i \notin[1,2 r] \cup[k-2 r+1, k]$.

Let $\mathcal{L}: D_{1} \cup D_{2} \rightarrow \Sigma_{\text {out }}$ assign output labels to $D_{1} \cup D_{2}$. We say that $\mathcal{L}$ is extendible w.r.t. $P$ if there exists a complete labeling $\mathcal{L}_{\diamond}$ of $P$ such that $\mathcal{L}_{\diamond}$ agrees with $\mathcal{L}$ on $D_{1} \cup D_{2}$, and $\mathcal{L}_{\diamond}$ is locally consistent at all nodes in $D_{2} \cup D_{3}$.

An Equivalence Class. We define an equivalence class $\stackrel{\star}{\sim}$ for the directed paths (i.e., the set of all non-empty strings in $\Sigma_{\text {in }}^{*}$ ), as follows.

Consider two directed paths $P=\left(u_{1}, \ldots, u_{x}\right)$ and $P^{\prime}=\left(v_{1}, \ldots, v_{y}\right)$, and let $\xi(P)=\left(D_{1}, D_{2}, D_{3}\right)$ and $\xi\left(P^{\prime}\right)=\left(D_{1}^{\prime}, D_{2}^{\prime}, D_{3}^{\prime}\right)$. Consider the following natural 1-to-1 correspondence $\phi:\left(D_{1} \cup D_{2}\right) \rightarrow$ $\left(D_{1}^{\prime} \cup D_{2}^{\prime}\right)$ defined as $\phi\left(u_{i}\right)=v_{i}$ and $\phi\left(u_{x-i+1}\right)=v_{y-i+1}$ for each $i \in[1,2 r]$. The 1-to-1 correspondence is well-defined so long as (i) $x=y$ or (ii) $x \geq 4 r$ and $y \geq 4 r$. We have $P \stackrel{\star}{\sim} P^{\prime}$ if and only if the following two statements are met:

- Isomorphism: The 1-to-1 correspondence $\phi$ is well-defined, and for each $u_{i} \in D_{1} \cup D_{2}$, the input label of $u_{i}$ is identical to the input label of $\phi\left(u_{i}\right)$.

- Extendibility: Let $\mathcal{L}$ be any assignment of output labels to nodes in $D_{1} \cup D_{2}$, and let $\mathcal{L}^{\prime}$ be the corresponding output labeling of $D_{1}^{\prime} \cup D_{2}^{\prime}$ under $\phi$. Then $\mathcal{L}$ is extendible w.r.t. $P$ if and only if $\mathcal{L}^{\prime}$ is extendible w.r.t. $P^{\prime}$.

Note that for the special case of $x \leq 4 r$, we have $P \stackrel{\star}{\sim} P^{\prime}$ if and only if $P$ is identical to $P^{\prime}$.

Define Type $(P)$ as the equivalence class of $P$ w.r.t. $\stackrel{\star}{\sim}$. The following technical lemma is analogous to [6, Lemma 1] in a specialized setting. We only use this lemma to prove the lemmas in Section 4.1. 
Lemma 10. Let $G$ be a path graph or a cycle graph where all nodes have input labels from $\Sigma_{\text {in }}$. Let $P$ be a directed subpath of $G$, and let $P^{\prime}$ be another directed path such that Type $\left(P^{\prime}\right)=\operatorname{Type}(P)$. We write $\xi(P)=\left(D_{1}, D_{2}, D_{3}\right)$ and $\xi\left(P^{\prime}\right)=\left(D_{1}^{\prime}, D_{2}^{\prime}, D_{3}^{\prime}\right)$. Let $\mathcal{L}_{\diamond}$ be any complete labeling of $G$ such that $\mathcal{L}_{\diamond}$ is locally consistent at all nodes in $D_{2} \cup D_{3}$. Let $G^{\prime}=\operatorname{Replace}\left(G, P, P^{\prime}\right)$ be the graph resulting from replacing $P$ with $P^{\prime}$ in $G$. Then there exists a complete labeling $\mathcal{L}_{\diamond}^{\prime}$ of $G^{\prime}$ such that the following two conditions are met.

1. For each $v \in(V(G) \backslash V(P)) \cup\left(D_{1} \cup D_{2}\right)$ and its corresponding $v^{\prime} \in\left(V\left(G^{\prime}\right) \backslash V\left(P^{\prime}\right)\right) \cup\left(D_{1}^{\prime} \cup D_{2}^{\prime}\right)$, we have $\mathcal{L}_{\diamond}(v)=\mathcal{L}_{\diamond}^{\prime}\left(v^{\prime}\right)$. Moreover, if $v \in(V(G) \backslash V(P)) \cup D_{1}$ and $\mathcal{L}_{\diamond}$ is locally consistent at $v$, then $\mathcal{L}_{\diamond}^{\prime}$ is locally consistent at $v^{\prime}$.

2. $\mathcal{L}_{\diamond}^{\prime}$ is locally consistent at all nodes in $D_{2}^{\prime} \cup D_{3}^{\prime}$.

Proof. The labeling $\mathcal{L}_{\diamond}^{\prime}\left(v^{\prime}\right)$ of $G^{\prime}$ for each $v^{\prime} \in\left(V\left(G^{\prime}\right) \backslash V\left(P^{\prime}\right)\right) \cup\left(D_{1}^{\prime} \cup D_{2}^{\prime}\right)$ is chosen "naturally" as follows. For each $v^{\prime} \in V\left(G^{\prime}\right) \backslash V\left(P^{\prime}\right)$, we set $\mathcal{L}_{\diamond}^{\prime}\left(v^{\prime}\right)=\mathcal{L}_{\diamond}(v)$ for its corresponding node $v \in V(G) \backslash V(P)$. For each $v^{\prime} \in D_{1}^{\prime} \cup D_{2}^{\prime}$, we set $\mathcal{L}_{\diamond}^{\prime}\left(v^{\prime}\right)=\mathcal{L}_{\diamond}(v)$ for its corresponding node $v \in D_{1} \cup D_{2}$ such that $\phi(v)=v^{\prime}$ in the definition of $\stackrel{\star}{\sim}$. At this point, it is clear that if $v \in(V(G) \backslash V(P)) \cup D_{1}$ has a locally consistent labeling under $\mathcal{L}_{\diamond}$, then its corresponding node $v^{\prime} \in\left(V\left(G^{\prime}\right) \backslash V\left(P^{\prime}\right)\right) \cup D_{1}^{\prime}$ also has a locally consistent labeling under $\mathcal{L}_{\diamond}^{\prime}$, so Condition 1 holds.

Now, the labeling $\mathcal{L}_{\diamond}^{\prime}$ is only undefined for nodes in $D_{3}^{\prime}$. We show that we can complete the labeling in such a way that is locally consistent at all nodes in $D_{2}^{\prime} \cup D_{3}^{\prime}$. Denote $\mathcal{L}$ as $\mathcal{L}_{\diamond}$ restricted to $D_{1} \cup D_{2}$. Since $\mathcal{L}_{\diamond}$ is locally consistent at all nodes in $P$, the labeling $\mathcal{L}$ is extendible w.r.t. $P$. Note that if we let $\mathcal{L}^{\prime}$ be $\mathcal{L}_{\diamond}$ restricted to $D_{1}^{\prime} \cup D_{2}^{\prime}$, then according to the way we define $\mathcal{L}_{\diamond}^{\prime}$, the two labeling $\mathcal{L}^{\prime}$ and $\mathcal{L}$ are identical under the 1 -to-1 correspondence $\phi$ specified in the definition of ${ }^{\star}$. That is, for each $v^{\prime} \in D_{1}^{\prime} \cup D_{2}^{\prime}$, we have $\mathcal{L}^{\prime}\left(v^{\prime}\right)=\mathcal{L}(v)$ for its corresponding node $v \in D_{1} \cup D_{2}$ such that $\phi(v)=v^{\prime}$. Since $P \stackrel{\star}{\sim} P^{\prime}$, the labeling $\mathcal{L}^{\prime}$ must be extendible w.r.t. $P^{\prime}$. That is, there is a way to assign $\mathcal{L}_{\diamond}^{\prime}\left(v^{\prime}\right)$ for each $v^{\prime} \in D_{3}^{\prime}$ such that all nodes in $D_{2}^{\prime} \cup D_{3}^{\prime}$ have locally consistent labelings under $\mathcal{L}_{\diamond}^{\prime}$, so Condition 2 holds.

One useful consequence of this lemma is that if we start with a path or a cycle $G$ with a legal labeling, after replacing its subpath $P$ with another one $P^{\prime}$ having the same type as $P$, then it is always possible to assign output labeling to $P^{\prime}$ to get a legal labeling without changing the already-assigned output labels of nodes outside of $P^{\prime}$.

Lemma 11. Let $G$ be a path graph or a cycle graph where all nodes have input labels from $\Sigma_{\mathrm{in}}$. Let $P$ be a directed subpath of $G$, and let $P^{\prime}$ be another directed path such that Type $\left(P^{\prime}\right)=\operatorname{Type}(P)$. Let $\mathcal{L}_{\diamond}$ be complete labeling of $G$ that is locally consistent at all nodes in $P$. Let $G^{\prime}=\operatorname{Replace}\left(G, P, P^{\prime}\right)$ be the graph resulting from replacing $P$ with $P^{\prime}$ in $G$. Then there exists a legal labeling $\mathcal{L}_{\diamond}^{\prime}$ of $G^{\prime}$ such that the following two conditions are met.

1. For each $v \in V(G) \backslash V(P)$ and its corresponding $v^{\prime} \in V\left(G^{\prime}\right) \backslash V\left(P^{\prime}\right)$, we have $\mathcal{L}_{\diamond}(v)=\mathcal{L}_{\diamond}^{\prime}\left(v^{\prime}\right)$. Moreover, if $\mathcal{L}_{\diamond}$ is locally consistent at $v \in V(G) \backslash V(P)$, then $\mathcal{L}_{\diamond}^{\prime}$ is locally consistent at $v^{\prime}$.

2. $\mathcal{L}_{\diamond}^{\prime}$ is locally consistent at all nodes in $P^{\prime}$.

Proof. We write $\xi\left(P^{\prime}\right)=\left(D_{1}^{\prime}, D_{2}^{\prime}, D_{3}^{\prime}\right)$. Condition 1 in this lemma is implied by Condition 1 in Lemma 10. To see that Condition 2 in this lemma holds, note that in this lemma we additionally require that $\mathcal{L}_{\diamond}$ is locally consistent at all nodes in $P$. Therefore, Condition 1 of Lemma 10 implies that $\mathcal{L}_{\diamond}^{\prime}$ is locally consistent at all nodes in $D_{1}^{\prime}$. This observation, together with Condition 2 of Lemma 10, implies that $\mathcal{L}_{\diamond}^{\prime}$ is locally consistent at all nodes in $P^{\prime}$. 
The following lemma is analogous to [6, Theorem 4] in a specialized setting. We only use this lemma in Section 4.1.

Lemma 12. Let $P=\left(v_{1}, \ldots, v_{k}\right)$, and let $P^{\prime}=\left(v_{1}, \ldots, v_{k-1}\right)$. Let the input label of $v_{k}$ be $\alpha$. Then Type $(P)$ is a function of $\alpha$ and Type $\left(P^{\prime}\right)$.

Proof. We prove the following stronger statement. Let $G$ be a directed path, and let $H$ be a directed subpath of $G$. Suppose $H^{\prime}$ is another directed path satisfying Type $(H)=\operatorname{Type}\left(H^{\prime}\right)$. Let $G^{\prime}=\operatorname{Replace}\left(G, H, H^{\prime}\right)$ be the result of replacing $H$ with $H^{\prime}$ in $G$. Then we claim that Type $(G)=\operatorname{Type}\left(G^{\prime}\right)$. The lemma is a corollary of this claim.

Consider the tripartitions $\xi(H)=\left(B_{1}, B_{2}, B_{3}\right), \xi\left(H^{\prime}\right)=\left(B_{1}^{\prime}, B_{2}^{\prime}, B_{3}^{\prime}\right), \xi(G)=\left(D_{1}, D_{2}, D_{3}\right)$, and $\xi\left(G^{\prime}\right)=\left(D_{1}^{\prime}, D_{2}^{\prime}, D_{3}^{\prime}\right)$. We write $B_{0}=V(G) \backslash V(H)$ and $B_{0}^{\prime}=V\left(G^{\prime}\right) \backslash V\left(H^{\prime}\right)$.

Let $\phi^{\star}$ be the natural 1-to-1 correspondence from $B_{0} \cup B_{1} \cup B_{2}$ to $B_{0}^{\prime} \cup B_{1}^{\prime} \cup B_{2}^{\prime}$. Note that $D_{1} \cup D_{2} \subseteq B_{0} \cup B_{1} \cup B_{2}$ and $D_{1}^{\prime} \cup D_{2}^{\prime} \subseteq B_{0}^{\prime} \cup B_{1}^{\prime} \cup B_{2}^{\prime}$. Also, the 1-to-1 correspondence between $D_{1} \cup D_{2}$ and $D_{1}^{\prime} \cup D_{2}^{\prime}$ given by $\phi^{\star}$ is exactly the 1 -to- 1 correspondence $\phi$ specified in the requirement of $G \stackrel{\star}{\sim} G^{\prime}$.

Let $\mathcal{L}:\left(D_{1} \cup D_{2}\right) \rightarrow \Sigma_{\text {out }}$ and let $\mathcal{L}^{\prime}$ be the corresponding output labeling of $D_{1}^{\prime} \cup D_{2}^{\prime}$, under the 1-to-1 correspondence $\phi$. To show that $G \stackrel{\star}{\sim} G^{\prime}$, all we need to do is show that $\mathcal{L}$ is extendible w.r.t. $G$ if and only if $\mathcal{L}^{\prime}$ is extendible w.r.t. $G^{\prime}$. Since we can also write $G=\operatorname{Replace}\left(G^{\prime}, H^{\prime}, H\right)$, it suffices to show just one direction, i.e., if $\mathcal{L}$ is extendible then $\mathcal{L}^{\prime}$ is extendible.

Suppose $\mathcal{L}$ is extendible. Then there exists an output labeling $\mathcal{L}_{\diamond}$ of $G$ such that (i) for each $v \in D_{1} \cup D_{2}$, we have $\mathcal{L}_{\diamond}(v)=\mathcal{L}(v)$, and (ii) $\mathcal{L}_{\diamond}$ is locally consistent at all nodes in $D_{2} \cup D_{3}$. Since $D_{2} \cup D_{3} \supseteq B_{2} \cup B_{3}$, we can apply Lemma 10, which shows that there exists a complete labeling $\mathcal{L}_{\diamond}^{\prime}$ of $G^{\prime}$ such that the two conditions in Lemma 10 are met. We argue that this implies that $\mathcal{L}^{\prime}$ is extendible. We verify that (i) $\mathcal{L}^{\prime}\left(v^{\prime}\right)=\mathcal{L}_{\diamond}^{\prime}\left(v^{\prime}\right)$ for each $v^{\prime} \in D_{1}^{\prime} \cup D_{2}^{\prime}$, and (ii) $\mathcal{L}_{\diamond}^{\prime}$ is locally consistent at all nodes in $D_{2}^{\prime} \cup D_{3}^{\prime}$.

- Condition 1 of Lemma 10 guarantees that $\mathcal{L}_{\diamond}(v)=\mathcal{L}_{\diamond}^{\prime}\left(\phi^{\star}(v)\right)$ for each $v \in(V(G) \backslash V(H)) \cup$ $\left(B_{1} \cup B_{2}\right)=B_{0} \cup B_{1} \cup B_{2}$ and its corresponding node $\phi^{\star}(v) \in B_{0}^{\prime} \cup B_{1}^{\prime} \cup B_{2}^{\prime}$. Since $D_{1}^{\prime} \cup D_{2}^{\prime} \subseteq$ $B_{0}^{\prime} \cup B_{1}^{\prime} \cup B_{2}^{\prime}$, we have $\mathcal{L}^{\prime}\left(v^{\prime}\right)=\mathcal{L}_{\diamond}^{\prime}\left(v^{\prime}\right)$ for each $v^{\prime} \in D_{1}^{\prime} \cup D_{2}^{\prime}$.

- The fact that $\mathcal{L}_{\diamond}$ is locally consistent at all nodes in $D_{2} \cup D_{3}$, together with Condition 1 in Lemma 10, guarantees that $\mathcal{L}_{\diamond}^{\prime}$ is locally consistent at all nodes in $\left(D_{2}^{\prime} \cup D_{3}^{\prime}\right) \backslash B_{3}^{\prime}$. Condition 2 in Lemma 10 guarantees that $\mathcal{L}_{\diamond}^{\prime}$ is locally consistent at all nodes in $B_{2}^{\prime} \cup B_{3}^{\prime}$. Therefore, $\mathcal{L}_{\diamond}^{\prime}$ is locally consistent at all nodes in $D_{2}^{\prime} \cup D_{3}^{\prime}$, as required.

The number of types can be upper bounded as follows.

Lemma 13. The number of equivalence classes of $\stackrel{\star}{\sim}$ (i.e., types) is at most $\left|\Sigma_{\text {in }}\right|^{4 r} 2^{\left|\Sigma_{\text {out }}\right|^{4 r}}$.

Proof. Let $P$ be a directed path, and let $\xi(P)=\left(D_{1}, D_{2}, D_{3}\right)$. Then Type $(P)$ is determined by the following information.

- The input labels in $D_{1} \cup D_{2}$. Note that there are at most $\left|\Sigma_{\text {in }}\right|^{4 r}$ possible input labeling of $D_{1} \cup D_{2}$.

- A length- $x$ binary string indicating the extendibility of each possible output labeling of $D_{1} \cup D_{2}$, where $x=\left|\Sigma_{\text {out }}\right|^{4 r}$.

Therefore, the number of equivalence classes of $\stackrel{\star}{\sim}$ is at most $\left.\left|\Sigma_{\text {in }}\right|{ }^{4 r} 2^{\mid \Sigma_{\text {out }}}\right|^{4 r}$. 
Define $\ell_{\text {pump }}$ as the total number of types. Observe that Lemma 12 implies that Type $(P)$ can be computed by a finite automaton whose number of states is the total number of types, which is a constant independent of $P$. Thus, we have the following two pumping lemmas which allow us to extend the length of a given directed path $P$ while preserving the type of $P$. The following two lemmas follow from the standard pumping lemma for regular language.

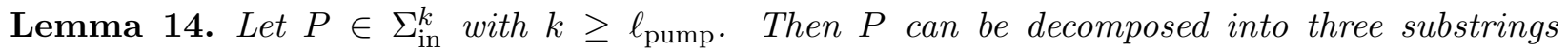
$P=x \circ y \circ z$ such that (i) $|x y| \leq \ell_{\text {pump, }}$, (ii) $|y| \geq 1$, and (iii) for each non-negative integer $i$, Type $\left(x \circ y^{i} \circ z\right)=\operatorname{Type}(P)$.

Lemma 15. For each $w \in \Sigma_{\mathrm{in}}^{>0}$, there exist two positive integers $a$ and $b$ such that $a+b \leq \ell_{\mathrm{pump}}$, and Type $\left(w^{a i+b}\right)$ is invariant for each non-negative integer $i$.

\subsection{The $\omega\left(\log ^{*} n\right)-o(n)$ Gap}

In this section we show that the $\omega\left(\log ^{*} n\right)-o(n)$ gap is decidable. More specifically, we show that an LCL problem $\mathcal{P}$ can be solved in $O\left(\log ^{*} n\right)$ rounds if and only if there exists a feasible function, which is defined as follows.

Input: A directed path $P=w_{1} \circ S \circ w_{2}$, where $\left|w_{1}\right| \in\left[\ell_{\text {pump }}, \ell_{\text {pump }}+1\right],\left|w_{2}\right| \in\left[\ell_{\text {pump }}, \ell_{\text {pump }}+1\right]$, and $|S|=2 r$. The decomposition $P=w_{1} \circ S \circ w_{2}$ is considered part of the input.

Output: A string $\mathcal{L} \in \Sigma_{\text {out }}^{2 r}$ that represents the output labeling of $S$.

Requirement: Any such function $f$ is said to be feasible if the following requirement is met for any paths $S_{1}, S_{2}$ and $w_{a}, w_{b}, w_{c}, w_{d}$ such that $\left|S_{1}\right|=\left|S_{2}\right|=2 r$ and $\left\{\left|w_{a}\right|,\left|w_{b}\right|,\left|w_{c}\right|,\left|w_{d}\right|\right\} \subseteq$ $\left[\ell_{\text {pump }}, \ell_{\text {pump }}+1\right]$. Let $P=w_{a} \circ S_{1} \circ w_{b} \circ w_{c} \circ S_{2} \circ w_{d}$, and consider the following assignment of output labels to $S_{1} \cup S_{2}$.

- Either label $S_{1}$ by $f\left(w_{a} \circ S_{1} \circ w_{b}\right)$ or label $S_{1}^{R}$ by $f\left(w_{b}^{R} \circ S_{1}^{R} \circ w_{a}^{R}\right)$.

- Either label $S_{2}$ by $f\left(w_{c} \circ S_{2} \circ w_{d}\right)$ or label $S_{2}^{R}$ by $f\left(w_{d}^{R} \circ S_{2}^{R} \circ w_{c}^{R}\right)$.

It is required that given such a partial labeling of $P$, the middle part $w_{b} \circ w_{c}$ can be assigned output labels in such a way that the labeling of (i) the last $r$ nodes of $S_{1}$, (ii) all nodes in $w_{b} \circ w_{c}$, and (iii) the first $r$ nodes of $S_{2}$ are locally consistent.

The following lemma is a straightforward consequence of the well-known $O\left(\log ^{*} n\right)$-round MIS algorithm on cycles.

Lemma 16. Let $G$ be a cycle graph of $n$ nodes, and let $s \leq k$ be two constant integers such that $s+k \leq n$. Then in $O\left(\log ^{*} n\right)$ rounds we can compute a decomposition $V=A \cup B$ such that each connected component of $A$ has size s, and each connected component of $B$ has size within $[k, k+1]$.

Proof. For any given constant integer $1 \leq L<n$, we will show that in $O\left(\log ^{*} n\right)$ time we can find an independent set $I$ of $G$ such that each connected component induced by $V \backslash I$ has at least $L$ nodes and at most $2 L$ nodes. Using this result with $L=2(s-1)+k(s+k+1)$, it is straightforward to obtain the desired decomposition $V=A \cup B$, as follows.

For each $v \in I$, it arbitrarily chooses a size- $s$ path $S_{v}$ that contains $v$, and all nodes in $S_{v}$ are included to $A$. Now each connected component $S^{\prime}$ induced by the remaining nodes is a path of size at least $L-2(s-1) \geq k(s+k+1)$ and at most $2 L$. We will divide the path $S^{\prime}$ into subpaths $R_{1}, R_{2}, \ldots, R_{t}$ meeting the following conditions: (i) if $i$ is odd, then the size of $R_{i}$ is $k$ or $k+1$; 
(ii) if $i$ is even, then the size of $R_{i}$ is $s$; (iii) $t$ is odd. Hence we obtain the desired decomposition $V=A \cup B$ if we include the nodes in $R_{1}, R_{3}, \ldots$ to $B$ and include the nodes in $R_{2}, R_{4}, \ldots$ to $A$. We show that such a decomposition of $S^{\prime}$ into subpaths $R_{1}, R_{2}, \ldots, R_{t}$ exists. Denote $z$ as the size of $S^{\prime}$. We write $z=\alpha(s+k+1)+\beta$, where $\alpha>0$ and $0 \leq \beta<s+k+1$ are integers. Note that we must have $\alpha \geq k$ and $\beta \leq 2 k$.

- For the case $\beta \geq k$, there is a decomposition $R_{1}, R_{2}, \ldots, R_{t}$ satisfies the following conditions: (i) if $i$ is odd, then the size of $R_{i}$ is $k+1$ when $i<t$ or $\beta$ when $i=t$; (ii) if $i$ is even, then the size of $R_{i}$ is $s$; (iii) $t=2 \alpha+1$ is odd.

- For the case $\beta<k$, there is a decomposition $R_{1}, R_{2}, \ldots, R_{t}$ satisfies the following conditions: (i) if $i$ is odd, then the size of $R_{i}$ is $k$ when $i \in\{1,3,5, \ldots, 2(k-\beta)-1\} \cup\{t\}$ or $k+1$ otherwise; (ii) if $i$ is even, then the size of $R_{i}$ is $s$; (iii) $t=2 \alpha+1$ is odd.

For the rest of the proof, we show that in $O\left(\log ^{*} n\right)$ time we can find the required independent set $I$. We prove the lemma by an induction on $L$. The base case of $L=1$ is identical to the MIS problem. Now consider $L>1$. By induction hypothesis, we find an independent set $I^{\prime}$ in $O\left(\log ^{*} n\right)$ time such that each connected component induced by $V \backslash I^{\prime}$ has at least $L^{\prime}$ nodes and at most $2 L^{\prime}$ nodes, where $L^{\prime}=\lfloor L / 2\rfloor$. Let $G^{\prime}$ be the graph resulting from contracting all nodes in $V \backslash I^{\prime}$, and we compute an MIS $I^{\prime \prime}$ on this graph $G^{\prime}$, which can be done in $O\left(\log ^{*} n\right)$ rounds in the original graph $G$. Note that each connected component $S$ of $V \backslash I^{\prime \prime}$ has size at least $2 L^{\prime}+1 \geq L$ and at most $3\left(2 L^{\prime}\right)+2$. If the size of $S$ is higher than $2 L$, then we can add some nodes in $S$ to the independent set $I^{\prime \prime}$ so that the component size of the remaining nodes in $S$ is within $[L, 2 L]$.

Lemma 17. If a feasible function $f$ exists, then there is an $O\left(\log ^{*} n\right)$-round deterministic LOCAL algorithm for $\mathcal{P}$ on cycles.

Proof. Given that the number of nodes $n$ is at least some large enough constant, in $O\left(\log ^{*} n\right)$ rounds we can compute a decomposition $V=A \cup B$ such that each connected component of $A$ has size $2 r$, and each connected component of $B$ has size within [ $\left.2 \ell_{\text {pump }}, 2 \ell_{\text {pump }}+1\right]$. This can be done using Lemma 16 with $s=2 r$ and $k=2 \ell_{\text {pump. }}$. We further decompose each connected component $P$ of $B$ into two paths $P=P_{1} \circ P_{2}$ in such a way that the size of both $P_{1}$ and $P_{2}$ are within the range $\left[\ell_{\text {pump }}, \ell_{\text {pump }}+1\right]$. We write $\mathscr{P}$ to denote the set of all these paths.

Let $S$ be a connected component of $A$, and let $w_{1}$ and $w_{2}$ be its two neighboring paths in $\mathscr{P}$ so that $\left(w_{1} \circ S \circ w_{2}\right)$ is a subpath of the underlying graph $G$. The output labels of $S$ are assigned either by labeling $S$ with $f\left(w_{1} \circ S \circ w_{2}\right)$ or by labeling $S^{R}$ with $f\left(w_{2}^{R} \circ S^{R} \circ w_{1}^{R}\right)$. At this moment, all components of $A$ have been assigned output labels using $f$. By the feasibility of $f$, each connected component of $B$ is able to label itself output labels in such a way that the labeling of all nodes are locally consistent.

Lemma 18. If there is an o(n)-round deterministic LOCAL algorithm $\mathcal{A}$ for $\mathcal{P}$ on cycles, then a feasible function $f$ exists.

Proof. Fix $s$ to be some sufficiently large number, and fix $n=8\left(s+\ell_{\text {pump }}\right)+2(2 r)$. We select $s$ to be large enough so that the runtime of $\mathcal{A}$ is smaller than $0.1 s$. For any given directed path $w$ with $|w| \in\left[\ell_{\text {pump }}, \ell_{\text {pump }}+1\right]$, we fix $w^{+}$as the result of applying the pumping lemma (Lemma 14) on $w$ so that the following two conditions are met: (i) $\left|w^{+}\right| \in\left[s, s+\ell_{\text {pump }}\right]$ and (ii) $\operatorname{Type}(w)=\operatorname{Type}\left(w^{+}\right)$. 
Constructing a Feasible Function $\boldsymbol{f}$ by Simulating $\mathcal{A}$. The function $f\left(w_{1} \circ S \circ w_{2}\right)$ is constructed by simulating a given $o(n)$-round deterministic LOCAL algorithm for $\mathcal{P}$. The output labeling given by $f\left(w_{1} \circ S \circ w_{2}\right)$ is exactly the result of simulating $\mathcal{A}$ on the path $P=w_{1}^{+} \circ S \circ w_{2}^{+}$ while assuming the number of nodes of the underlying graph is $n$. Remember that the round complexity of $\mathcal{A}$ is $o(n)$ on $n$-node graphs. By setting $s$ to be large enough, the runtime of $\mathcal{A}$ can be made smaller than $0.1 s$. Thus, the calculation of $f\left(w_{1} \circ S \circ w_{2}\right)$ only depends on the IDs and the input labels of (i) the last $0.1 s$ nodes in $w_{1}^{+}$, (ii) all nodes in $S$, and (iii) the first $0.1 s$ nodes in $w_{2}^{+}$. In the calculation of $f\left(w_{1} \circ S \circ w_{2}\right)$, the IDs of the nodes that participate in the simulation of $\mathcal{A}$ are chosen arbitrarily so long as they are distinct.

Feasibility of $f$. Now we verify that the function $f$ constructed above is feasible. Consider any choices of paths $S_{1}, S_{2}$ and $w_{a}, w_{b}, w_{c}, w_{d}$ such that $\left|S_{1}\right|=\left|S_{2}\right|=2 r$ and $\left\{\left|w_{a}\right|,\left|w_{b}\right|,\left|w_{c}\right|,\left|w_{d}\right|\right\} \subseteq$ $\left[\ell_{\text {pump }}, \ell_{\text {pump }}+1\right]$. Define $P=w_{a} \circ S_{1} \circ w_{b} \circ w_{c} \circ S_{2} \circ w_{d}$, and let $G$ be the cycle graph formed by connecting the two ends of the path $P$. To show that $f$ is feasible, we need to consider the following four ways of assigning output labels to $S_{1} \cup S_{2}$.

1. Label $S_{1}$ by $f\left(w_{a} \circ S_{1} \circ w_{b}\right)$; label $S_{2}$ by $f\left(w_{c} \circ S_{2} \circ w_{d}\right)$.

2. Label $S_{1}$ by $f\left(w_{a} \circ S_{1} \circ w_{b}\right)$; label $S_{2}^{R}$ by $f\left(w_{d}^{R} \circ S_{2}^{R} \circ w_{c}^{R}\right)$.

3. Label $S_{1}^{R}$ by $f\left(w_{b}^{R} \circ S_{1}^{R} \circ w_{a}^{R}\right)$; label $S_{2}$ by $f\left(w_{c} \circ S_{2} \circ w_{d}\right)$.

4. Label $S_{1}^{R}$ by $f\left(w_{b}^{R} \circ S_{1}^{R} \circ w_{a}^{R}\right)$; label $S_{2}^{R}$ by $f\left(w_{d}^{R} \circ S_{2}^{R} \circ w_{c}^{R}\right)$.

For each of the above four partial labelings of $P$, we need to show that the middle part $w_{b} \circ w_{c}$ can still be assigned output labels in such a way that the labeling of (i) the last $r$ nodes of $S_{1}$, (ii) all nodes in $w_{b} \circ w_{c}$, and (iii) the first $r$ nodes of $S_{2}$ are locally consistent.

Proof of the First Case. In what follows, we focus on the first case, i.e., the partial labeling is given by labeling $S_{1}$ by $f\left(w_{a} \circ S_{1} \circ w_{b}\right)$ and labeling $S_{2}$ by $f\left(w_{c} \circ S_{2} \circ w_{d}\right)$; the proof for the other three cases are analogous. In this case, we define $P^{\prime}=w_{a}^{+} \circ S_{1} \circ w_{b}^{+} \circ w_{c}^{+} \circ S_{2} \circ w_{d}^{+}$, and let $G^{\prime}$ be the cycle graph formed by connecting the two ends of $P^{\prime}$. Note that the number of nodes in $G^{\prime}$ is at most $8\left(s+\ell_{\text {pump }}\right)+2(2 r)=n$. All we need to do is to find an output labeling $\mathcal{L}$ of $G$ such that the following conditions are satisfied.

(a) The output labels of $S_{1}$ is given by $f\left(w_{a} \circ S_{1} \circ w_{b}\right)$.

(b) The output labels of $S_{2}$ is given by $f\left(w_{c} \circ S_{2} \circ w_{d}\right)$.

(c) The labeling of (i) the last $r$ nodes of $S_{1}$, (ii) all nodes in $w_{b} \circ w_{c}$, and (iii) the first $r$ nodes of $S_{2}$ are locally consistent.

We first generate an output labeling $\mathcal{L}^{\prime}$ of $G^{\prime}$ by executing $\mathcal{A}$ on $G^{\prime}$ under the following ID assignment. The IDs of (i) the last $0.1 s$ nodes in $w_{a}^{+}$, (ii) all nodes in $S_{1}$, and (iii) the first $0.1 s$ nodes in $w_{b}^{+}$are chosen as the ones used in the definition of $f\left(w_{a} \circ S_{1} \circ w_{b}\right)$. Similarly, the IDs of (i) the last $0.1 s$ nodes in $w_{c}^{+}$, (ii) all nodes in $S_{2}$, and (iii) the first $0.1 s$ nodes in $w_{d}^{+}$are chosen as the ones used in the definition of $f\left(w_{c} \circ S_{2} \circ w_{d}\right)$. The IDs of the rest of the nodes are chosen arbitrarily so long as when we run $\mathcal{A}$ on $G^{\prime}$, no node sees two nodes with the same ID. Due to the way we define $f$, the output labeling $\mathcal{L}^{\prime}$ of the subpath $S_{1}$ is exactly given by $f\left(w_{a} \circ S_{1} \circ w_{b}\right)$, and the output labeling $\mathcal{L}^{\prime}$ of $S_{2}$ is exactly $f\left(w_{c} \circ S_{2} \circ w_{d}\right)$. Due to the correctness of $\mathcal{A}, \mathcal{L}^{\prime}$ is a legal labeling.

We transform the output labeling $\mathcal{L}^{\prime}$ of $G^{\prime}$ to a desired output labeling $\mathcal{L}$ of $G$. Remember that $G$ is the result of replacing the four subpaths $w^{+}$of $G^{\prime}$ by $w$, and we have $\operatorname{Type}\left(w^{+}\right)=\operatorname{Type}(w)$. In view of Lemma 11, there is a legal labeling $\mathcal{L}$ of $G$ such that all nodes in $S_{1}$ and $S_{2}$ are labeled the same as in $G^{\prime}$. Therefore, the labeling $\mathcal{L}$ satisfies the above three conditions (a), (b), and (c). 
The Other Cases. We briefly discuss how we modify the proof to deal with the other three cases. For example, consider the second case, where the partial labeling is given by labeling $S_{1}$ by $f\left(w_{a} \circ S_{1} \circ w_{b}\right)$ and labeling $S_{2}^{R}$ by $f\left(w_{d}^{R} \circ S_{2}^{R} \circ w_{c}^{R}\right)$. In this case, the path $P^{\prime}$ is defined as

$$
P^{\prime}=w_{a}^{+} \circ S_{1} \circ w_{b}^{+} \circ\left(\left(w_{c}^{R}\right)^{+}\right)^{R} \circ S_{2}^{R} \circ\left(\left(w_{d}^{R}\right)^{+}\right)^{R} .
$$

During the ID assignment of $G^{\prime}$, the IDs of (i) the last $0.1 s$ nodes in $w_{c}^{+}$, (ii) all nodes in $S_{2}$, and (iii) the first $0.1 s$ nodes in $w_{d}^{+}$are now chosen as the ones used in the definition of $f\left(w_{d}^{R} \circ S_{2}^{R} \circ w_{c}^{R}\right)$. Using such an ID assignment, the output labeling $\mathcal{L}^{\prime}$ of $S_{2}^{R}$ as the result of executing $\mathcal{A}$ on $G^{\prime}$ will be exactly the same as the output labeling given by $f\left(w_{d}^{R} \circ S_{2}^{R} \circ w_{c}^{R}\right)$. The rest of the proof is the same.

Theorem 8 follows from the above two lemmas. The decidability result is due to the simple observation that whether a feasible function exists is decidable.

\subsection{Partitioning a Cycle}

In the following sections, we prove the decidability result associated with the $\omega(1)-o\left(\log ^{*} n\right)$ gap. In this proof, we also define a feasible function, prove its decidability, and show the existence given an $o\left(\log ^{*} n\right)$-time algorithm. The main challenge here is that an MIS cannot be computed in $O(1)$ time. To solve this issue, we decompose a cycle into paths with unrepetitive patterns and paths with repetitive patterns. For paths with unrepetitive patterns, we are able to compute a sufficiently well-spaced MIS in $O(1)$ time by making use of the irregularity of the input patterns.

Section 4.3 considers an $O(1)$-round algorithm that partitions a cycle into some short paths and some paths that have a repeated input pattern. Section 4.4 defines a feasible function whose existence characterizes the $O(1)$-round solvable LCL problems. In Section 4.5, we prove Theorem 9.

Partitioning an Undirected Cycle into Directed Paths. Let $G$ be a cycle graph. An orientation of a node $v$ is an assignment to one of its neighbor, this can be specified using portnumbering. An orientation of the nodes in $G$ is called $\ell$-orientation if the following condition is met. If $|V(G)| \leq \ell$, then all nodes in $G$ are oriented to the same direction. If $|V(G)|>\ell$, then each node $v \in V(G)$ belongs to a path $P$ such that (i) all nodes in $P$ are oriented to the same direction, and (ii) the number of nodes in $P$ is at least $\ell$. In $O(1)$ rounds we can compute an $\ell$-orientation of $G$ for any constant $\ell$.

Lemma 19 ([6]). Let $G$ be a cycle graph. Let $\ell$ be a constant. There is a deterministic LOCAL algorithm that computes an $\ell$-orientation of $G$ in $O(1)$ rounds.

In this section, we will use a generalization of an $\ell$-orientation that satisfies an additional requirement that the input labels of each directed path $P$ in the decomposition with $|V(P)|>2 \ell_{\text {width }}$ (where $2 \ell_{\text {width }}$ is a threshold) must form a periodic string (whose period length is at most $\ell_{\text {pattern }}$ ).

A string $w \in \Sigma_{\text {in }}^{*}$ is called primitive if $w$ cannot be written as $x^{i}$ for some $x \in \Sigma_{\text {in }}^{*}$ and $i \geq 2$. Let $G$ be a cycle graph or a path graph where each node $v \in V(G)$ has an input label from $\Sigma_{\text {in. }}$. We define an $\left(\ell_{\text {width }}, \ell_{\text {count }}, \ell_{\text {pattern }}\right)$-partition as a partition of $G$ into a set of connected subgraphs $\mathscr{P}$ meeting the following criteria. We assume $|V(G)|>2 \ell_{\text {width }}$ and $\ell_{\text {pattern }} \geq \ell_{\text {width }}$.

Direction and Minimum Length: For each $P \in \mathscr{P}$, the nodes in $P$ are oriented to the same direction, and $|V(P)| \geq \ell_{\text {width }}$. 
Short Paths: Define $\mathscr{P}_{\text {short }}$ as the subset of $\mathscr{P}$ that contains paths having at most $2 \ell_{\text {width }}$ nodes. For each directed path $P=\left(v_{1}, \ldots, v_{k}\right) \in \mathscr{P}_{\text {short }}$, each node $v_{i}$ in $P$ knows its rank $i$.

Long Paths: Define $\mathscr{P}_{\text {long }}=\mathscr{P} \backslash \mathscr{P}_{\text {short }}$. Then the input labeling of the nodes in $P$ is of the form $w^{k}$ for some primitive string $w \in \Sigma_{\text {in }}^{*}$ such that $|w| \leq \ell_{\text {pattern }}$ and $k \geq \ell_{\text {count }}$. Moreover, each node $v$ in $P$ knows the string $w$.

Note that $\mathscr{P}$ may contain a cycle. This is possible only when $G$ is a cycle where the input labeling is a repetition (at least $\ell_{\text {count }}$ times) of a primitive string $w \in \Sigma_{\text {in }}^{*}$ of length at most $\ell_{\text {pattern }}$. In this case, we must have $\mathscr{P}=\mathscr{P}_{\text {long }}=\{G\}$. Otherwise, $\mathscr{P}$ contains only paths.

The goal of this section is to show that an $\left(\ell_{\text {width }}, \ell_{\text {count }}, \ell_{\text {pattern }}\right)$-partition can be found in $O(1)$ rounds. First of all, in Lemma 20 we demonstrate how we can break symmetry in $O(1)$ rounds given that the underlying graph is directed and the input labels does not form long periodic strings. Let $G$ be a path or a cycle. A set $I \subseteq V(G)$ is called an $(\alpha, \beta)$-independent set if the following conditions are met: (i) $I$ is an independent set, and $I$ does not contain either endpoint of $G$ (if $G$ is a path), and (ii) each connected component induced by $V \backslash I$ has at least $\alpha$ nodes and at most $\beta$ nodes, unless $|V| \leq \alpha$, in which case we allow $I=\emptyset$. Note that finding an $(\alpha, \beta)$-independent set takes $O\left(\log ^{*} n\right)$ rounds in general, but in Lemma 20 we show that by leveraging the "irregularity" of input labels, we can do this in $O(1)$ rounds on directed paths or cycles without periodic patterns.

Lemma 20. Let $\gamma$ and $\ell$ be any two constants with $\ell \geq \gamma$. Let $G$ be a directed cycle or a directed path that does not contain any subpath of the form $w^{x}$, with $|w| \leq \gamma$ and $\left|w^{x}\right| \geq \ell$. There is a deterministic LOCAL algorithm that computes an $(\gamma, 2 \gamma)$-independent set $I$ of $G$ in $O(1)$ rounds.

Proof. For the case $G$ is a directed path $P=(s, \ldots, t)$, define $V^{\prime}$ as the set of nodes in $G$ whose distance to $t$ is at least $\ell-1$. For the case $G$ is a directed cycle, define $V^{\prime}=V(G)$. In what follows, we focus on finding an $(\gamma, 2 \gamma)$-independent set $I^{\prime}$ of the nodes in $V^{\prime}$. Extending the set $I^{\prime}$ to produce the desired independent set $I$ can be done with extra $O(1)$ rounds.

Recall that $G$ is directed. Define the color of a node $v \in V^{\prime}$ by the sequence of the $\ell$ input labels of $v$ and the $\ell-1$ nodes following $v$ in $G$. For each node $v \in V^{\prime}$, there is no other node within distance $\gamma$ to $v$ having the same color as $v$, since otherwise we can find a subpath whose input labels form a string $w^{x}$, with $|w| \leq \gamma$ and $\left|w^{x}\right| \geq \ell$. By applying the standard procedure that computes an MIS from a coloring, within $O(1)$ rounds a $(\gamma, 2 \gamma)$-independent set $I^{\prime}$ can be obtained.

Using Lemma 20, we first show that an $\left(\ell_{\text {width }}, \ell_{\text {count }}, \ell_{\text {pattern }}\right)$-partition can be found in $O(1)$ rounds for the case $G$ is directed. That is, all nodes in $G$ are initially oriented to the same direction, and we are allowed to re-orient the nodes.

Lemma 21. Let $G$ be a directed cycle or a directed path where each node $v \in V(G)$ has an input label from $\Sigma_{\mathrm{in}}$, and $|V(G)|>2 \ell_{\text {width }}$. Let $\ell_{\text {width }}, \ell_{\text {count }}, \ell_{\text {pattern }}$ be three constants such that $\ell_{\text {pattern }} \geq \ell_{\text {width }}$. There is a deterministic LOCAL algorithm that computes an $\left(\ell_{\text {width }}, \ell_{\text {count }}, \ell_{\text {pattern }}\right)$-partition in $O(1)$ rounds

Proof. Let $\left(w_{1}, w_{2}, \ldots, w_{k}\right)$ be any ordering of the primitive strings in $\Sigma_{\text {in }}^{*}$ of length at most $\ell_{\text {pattern }}$. First, construct a set of subgraphs $\mathscr{P}_{\text {long }}$ as follows. Initialize $U=V(G)$ and $\mathscr{P}_{\text {long }}=\emptyset$. For $i=1$ to $k$, execute the following procedure. Let $S_{i}$ be the set of maximal-size connected subgraphs formed by nodes in $U$ such that the input labels form the string $w_{i}^{x}$ with $x \geq \ell_{\text {count }}+2 \ell_{\text {width }}$. Each node $v \in U$ in $O(1)$ rounds checks if $v$ belongs to a subgraph in $S_{i}$; if so, remove $v$ from $U$. For each $P \in S_{i}$, 
define $P^{\prime}$ as follows. If $P$ is a cycle, then $P^{\prime}=P$. If $P$ is a path, then $P^{\prime}$ is the result of removing all nodes that are within distance $\ell_{\text {width }}\left|w_{i}\right|-1$ to an endpoint in $P$. Note that each node $v$ in $P$ knows whether $v$ belongs to $P^{\prime}$. Define $S_{i}^{\prime}=\left\{P^{\prime} \mid P \in S_{i}\right\}$, and then update $\mathscr{P}_{\text {long }} \leftarrow \mathscr{P}_{\text {long }} \cup S_{i}^{\prime}$.

It is straightforward to verify that each path or cycle $P \in \mathscr{P}_{\text {long }}$ satisfies the requirement in the definition of $\left(\ell_{\text {width }}, \ell_{\text {count }}, \ell_{\text {pattern }}\right)$-partition. Define the set of subgraphs $\mathscr{P}_{\text {irreg }}$ as the connected components of the nodes not in any subgraph in $\mathscr{P}_{\text {long }}$. Define $\ell=\left(\ell_{\text {pattern }}+2 \ell_{\text {width }}\right) \cdot \ell_{\text {count }}$. By our construction, the input labeling in each subgraph $P \in \mathscr{P}_{\text {irreg }}$ does not contain any substring $w^{x}$, with $1 \leq|w| \leq \ell_{\text {pattern }}$ and $\left|w^{x}\right| \geq \ell$. An $\left(\ell_{\text {pattern }}, 2 \ell_{\text {pattern }}\right)$-independent set of each $P \in \mathscr{P}$ irreg can be computed using Lemma 20 in $O(1)$ rounds. Observe that each subgraph $P \in \mathscr{P}_{\text {irreg }}$ has at least $\ell_{\text {width }}$ nodes. Given an $\left(\ell_{\text {pattern }}, 2 \ell_{\text {pattern }}\right)$-independent set of a subgraph $P \in \mathscr{P}_{\text {irreg }}$, in $O(1)$ rounds $P$ can be partitioned into subpaths, each of which contains at least $\ell_{\text {pattern }}$ nodes and at most $2 \ell_{\text {pattern }}$ nodes. This finishes the construction of an $\left(\ell_{\text {width }}, \ell_{\text {count }}, \ell_{\text {pattern }}\right)$-partition.

Combining Lemma 21 and Lemma 19, we are able to construct an $\left(\ell_{\text {width }}, \ell_{\text {count }}, \ell_{\text {pattern }}\right)$-partition in $O(1)$ rounds for undirected graphs.

Lemma 22. Let $G$ be a cycle or a path where each node $v \in V(G)$ has an input label from $\Sigma_{\mathrm{in}}$, and $|V(G)|>2 \ell_{\text {width }}$. Let $\ell_{\text {width }}, \ell_{\text {count }}, \ell_{\text {pattern }}$ be three constants such that $\ell_{\text {pattern }} \geq \ell_{\text {width }}$. There is a deterministic LOCAL algorithm that computes an $\left(\ell_{\text {width }}, \ell_{\text {count }}, \ell_{\text {pattern }}\right)$-partition in $O(1)$ rounds

Proof. The algorithm is as follows. Compute an $\ell$-orientation of $G$ by Lemma 19 in $O(1)$ rounds with $\ell=2 \ell_{\text {width }}+1$. For each maximal-length connected subgraph $P$ where each constituent node is oriented to the same direction, find an $\left(\ell_{\text {width }}, \ell_{\text {count }}, \ell_{\text {pattern }}\right)$-partition of $P$ in $O(1)$ rounds by Lemma 21.

\subsection{Feasible Function}

The goal of this section is to define a feasible function whose existence characterizes the $O(1)$-round solvable $\mathrm{LCL}$ problems. With respect to an $\mathrm{LCL}$ problem $\mathcal{P}$ and a function $f$ which takes a string $w \in \Sigma_{\text {in }}^{k}$ with $1 \leq k \leq \ell_{\text {pump }}$ as input, and returns a string $f(w) \in \Sigma_{\text {out }}^{k}$, we define some partially or completely labeled path graphs which are used in the definition of a feasible function.

Completely Labeled Graph $\mathcal{G}_{\boldsymbol{w}, \boldsymbol{z}}$ : Let $w \in \Sigma_{\text {in }}^{*}$ be any string of length at least 1 and at most $\ell_{\text {pump. }}$. Let $z$ be any non-negative integer. Define $\mathcal{G}_{w, z}=\left(G_{w, z}, \mathcal{L}\right)$ as follows. The graph $G_{w, z}$ is a path of the form $w^{r} \circ w^{z} \circ w^{r}$. The labeling $\mathcal{L}$ is a complete labeling of the form $f(w)^{z+2 r}$. Define $\operatorname{Mid}\left(G_{w, z}\right)$ as the middle subpath $w^{z}$ of $G_{w, z}$.

Partially Labeled Graph $\mathcal{G}_{\boldsymbol{w}_{1}, \boldsymbol{w}_{\mathbf{2}}, \boldsymbol{S}}$ : Let $w_{1}, w_{2} \in \Sigma_{\text {in }}^{*}$ be any two strings of length at least 1 and at most $\ell_{\text {pump. }}$ Let $S \in \Sigma_{\text {in }}^{*}$ be any string (can be empty). Define $\mathcal{G}_{w_{1}, w_{2}, S}=\left(G_{w_{1}, w_{2}, S}, \mathcal{L}\right)$ as follows. The graph $G_{w_{1}, w_{2}, S}$ is the path of the form $w_{1}^{\ell \text { pump }+2 r} \circ S \circ w_{2}^{\ell \text { pump }}+2 r$. The labeling $\mathcal{L}$ is a partial labeling of $G_{w_{1}, w_{2}, S}$ which fixes the output labels of the first $2 r\left|w_{1}\right|$ and the last $2 r\left|w_{2}\right|$ nodes by $f\left(w_{1}\right)^{2 r}$ and $f\left(w_{2}\right)^{2 r}$, respectively. Define $\operatorname{Mid}\left(G_{w_{1}, w_{2}, S}\right)$ as the middle subpath $w_{1}^{\ell_{\text {pump }}+r} \circ S \circ w_{2}^{\ell_{\text {pump }}+r}$ of $G_{w_{1}, w_{2}, S}$.

Feasible Function: We call $f$ a feasible function if the following conditions are met: (i) For each $\mathcal{G}_{w, z}=\left(G_{w, z}, \mathcal{L}\right)$, the complete labeling $\mathcal{L}$ is locally consistent at all nodes in $\operatorname{Mid}\left(G_{w, z}\right)$. (ii) Each partially labeled graph $\mathcal{G}_{w_{1}, w_{2}, S}$ admits a complete labeling $\mathcal{L}_{\diamond}$ that is locally consistent at all nodes in $\operatorname{Mid}\left(\mathcal{G}_{w_{1}, w_{2}, S}\right)$.

Lemma 23. Given an $\mathrm{LCL}$ problem $\mathcal{P}$ on cycle graphs. It is decidable whether there is a feasible function. 
Proof. Note that it is not immediate from its definition as to whether a feasible function exists is decidable, since there appears to be infinitely many graphs $\mathcal{G}_{w, z}$ and $\mathcal{G}_{w_{1}, w_{2}, S}$ needed to be examined. However, the following simple observations show that it suffices to check only a constant number of these graphs.

- If the complete labeling $\mathcal{L}$ of $\mathcal{G}_{w, 1}=\left(G_{w, 1}, \mathcal{L}\right)$ is locally consistent at all nodes in $\operatorname{Mid}\left(G_{w, 1}\right)$, then for all $z \geq 1$, the complete labeling $\mathcal{L}$ of $\mathcal{G}_{w, z}=\left(G_{w, z}, \mathcal{L}\right)$ is also locally consistent at all nodes in $\operatorname{Mid}\left(G_{w, z}\right)$.

- If $\mathcal{G}_{w_{1}, w_{2}, S}$ admits a complete labeling $\mathcal{L}_{\diamond}$ that is locally consistent at all nodes in $\operatorname{Mid}\left(\mathcal{G}_{w_{1}, w_{2}, S}\right)$, then for each $S^{\prime}$ such that $\operatorname{Type}(S)=\operatorname{Type}\left(S^{\prime}\right)$, the partially labeled graph $\mathcal{G}_{w_{1}, w_{2}, S^{\prime}}$ also admits a complete labeling $\mathcal{L}_{\diamond}$ that is locally consistent at all nodes in $\operatorname{Mid}\left(\mathcal{G}_{w_{1}, w_{2}, S^{\prime}}\right)$. This is due to Lemma 11.

Therefore, to decide whether a function $f$ is feasible, we only need to check all possible $\mathcal{G}_{w, z}$ and $\mathcal{G}_{w_{1}, w_{2}, S}$. For each $w$ we only need to consider the graph $\mathcal{G}_{w, z}$ with $z=1$. For each $w_{1}$ and $w_{2}$, we do not need to go over all $S$; we only need to consider (i) the empty string $S=\emptyset$, and (ii) for each type $\tau$, a string $S \in \Sigma_{\text {in }}^{*}$ such that Type $(S)=\tau$. By Lemma 14, for each type $\tau$, there exists $P \in \Sigma_{\text {in }}^{x}$ with $x \leq \ell_{\text {pump }}$ such that Type $(P)=\tau$. Therefore, a string $S$ with $\operatorname{Type}(S)=\tau$ can be found in bounded amount of time; also note that the number of types is bounded; see Lemma 13.

For the rest of this section, we show that as long as the deterministic LOCAL complexity of $\mathcal{P}$ is $o\left(\log ^{*} n\right)$ on cycle graphs, there exists a feasible function $f$. In Lemma 24 we show how to extract a function $f$ from a given $o\left(\log ^{*} n\right)$-round deterministic LOCAL algorithm $\mathcal{A}$, and then in Lemma 25 we prove that such a function $f$ is feasible. Intuitively, Lemma 24 shows that there exists an ID-assignment such that when we run $\mathcal{A}$ on a subpath whose input labeling is a repetition of a length- $k$ pattern $w$, the output labeling is also a repetition of a length- $k$ pattern $w^{\prime}$. The function $f$ will be defined as $f(w)=w^{\prime}$.

Lemma 24. Let $\mathcal{A}$ be any deterministic LOCAL algorithm that solves $\mathcal{P}$ in $t(n)=o\left(\log ^{*} n\right)$ rounds. Then there is a number $n^{\prime}$ and function $f$ which takes a string $w \in \Sigma_{\text {in }}^{k}$ with $1 \leq k \leq \ell_{\text {pump }}$ as input, and returns a string $f(w) \in \Sigma_{\text {out }}^{k}$ meeting the following condition. For any $P=w^{i} \circ w^{2 r+1} \circ w^{i}$ such that $\left|w^{i}\right| \geq t\left(n^{\prime}\right)$ and $1 \leq|w| \leq \ell_{\text {pump }}$, there is an assignment of distinct $\Theta\left(\log n^{\prime}\right)$-bit IDs to the nodes in $P$ such that the following is true. Simulating $\mathcal{A}$ on $P$ while assuming that the total number of nodes in the underlying graph is $n^{\prime}$ yields the output labeling $f(w)^{2 r+1}$ for the middle subpath $w^{2 r+1}$.

Proof. In this proof we assume that there is no such a number $n^{\prime}$. Then we claim that using $\mathcal{A}$ it is possible to obtain a deterministic LOCAL algorithm for MIS on an n-node directed cycle $G$ without input labeling, in $O(t(n))+O(1)=o\left(\log ^{*} n\right)$ rounds. This contradicts the well-known $\Omega\left(\log ^{*} n\right)$ lower bound for MIS [20].

Let $G$ be an $n$-node directed cycle without input labeling. The MIS algorithm on $G$ is described as follows. Let $w \in \Sigma_{\text {in }}^{k}$ with $1 \leq k \leq \ell_{\text {pump }}$ be chosen such that for any function $f$, the string $f(w) \in \Sigma_{\text {out }}^{k}$ does not satisfy the conditions stated in the lemma for the number $n^{\prime}=n k$. Define $G^{\prime}$ as the graph resulting from replacing each node $v \in V(G)$ with a path $w$. We can simulate the imaginary graph $G^{\prime}$ in the communication network $G$ by letting each node $v \in V(G)$ simulate a path $w$.

We execute the algorithm $\mathcal{A}$ on $G^{\prime}$ while assuming that the total number of nodes is $n^{\prime}$. The execution takes $t\left(n^{\prime}\right)=O(t(n))$ rounds. For each node $v \in V(G)$, define the color of $v$ as the sequence of the output labels of the path $w^{2 r}$ simulated by the node $v$ and the $2 r-1$ nodes following 
$v$ in the directed cycle $G$. This gives us a proper $O(1)$-coloring, since otherwise there must exist a subpath $P=w^{2 r+1}$ of $G^{\prime}$ such that the output labeling of $P$ is of the form $y^{2 r+1}$ for some $y$, contradicting our choice of $w$. Using the standard procedure of computing an MIS from a coloring, with extra $O(1)$ rounds, an MIS of $G$ can be obtained.

Note that there is a subtle issue about how we set the IDs of nodes in $V\left(G^{\prime}\right)$. The following method is guaranteed to output distinct IDs. Let $v \in V(G)$, and let $u_{1}, \ldots, u_{k}$ be the nodes in $V\left(G^{\prime}\right)$ simulated by $v$. Then we may use $\operatorname{ID}\left(u_{i}\right)=k \cdot \operatorname{ID}(v)+i$.

Lemma 25. Suppose that the deterministic LOCAL complexity of $\mathcal{P}$ is o $\left(\log ^{*} n\right)$ on cycle graphs. Then there exists a feasible function $f$.

Proof. Let $\mathcal{A}$ be any deterministic LOCAL algorithm that solves $\mathcal{P}$ in $t(n)=o\left(\log ^{*} n\right)$ rounds. Let $n^{\prime}$ and $f$ be chosen to meet the conditions in Lemma 24 for $\mathcal{A}$. The goal of the proof is to show that $f$ is a feasible function. According to the conditions specified in Lemma 24 for the function $f$, we already know that the complete labeling $\mathcal{L}$ of each $\mathcal{G}_{w, z}=\left(G_{w, z}, \mathcal{L}\right)$ is locally consistent at all nodes in $\operatorname{Mid}\left(G_{w, z}\right)$. Therefore, all we need to do is the following. For each partially labeled graph $\mathcal{G}_{w_{1}, w_{2}, S}$, find a complete labeling $\mathcal{L}_{\diamond}$ that is locally consistent at all nodes in $\operatorname{Mid}\left(\mathcal{G}_{w_{1}, w_{2}, S}\right)$.

Given the three parameters $w_{1}, w_{2}$, and $S$, define $G$ as the cycle resulting from linking the two ends of the path $w_{1}^{\ell_{\text {pump }}} \circ w_{1}^{2 r+1} \circ w_{1}^{\ell_{\text {pump }}} \circ S \circ w_{2}^{\ell_{\text {pump }}} \circ w_{2}^{2 r+1} \circ w_{2}^{\ell_{\text {pump }}}$. Define $\mathcal{L}$ as the partial labeling of $G$ which fixes the output labeling of the two subpaths $w_{1}^{2 r+1}$ and $w_{2}^{2 r+1}$ by $f\left(w_{1}\right)^{2 r+1}$ and $f\left(w_{2}\right)^{2 r+1}$, respectively. We write $P_{1}^{\text {mid }}$ and $P_{2}^{\text {mid }}$ to denote the two subpaths $w_{1}^{2 r+1}$ and $w_{2}^{2 r+1}$, respectively.

In what follows, we show that the partially labeled graph $\mathcal{G}=(G, \mathcal{L})$ admits a legal labeling $\mathcal{L}_{\diamond}$. Since $\mathcal{G}_{w_{1}, w_{2}, S}$ is a subgraph of $\mathcal{G}=(G, \mathcal{L})$, such a legal labeling $\mathcal{L}_{\diamond}$ is also a complete labeling of $\mathcal{G}_{w_{1}, w_{2}, S}$ that is locally consistent at all nodes in $\operatorname{Mid}\left(\mathcal{G}_{w_{1}, w_{2}, S}\right)$.

For the rest of the proof, we show the existence of $\mathcal{L}_{\diamond}$. This will be established by applying a pumping lemma. Define the graph $G^{\prime}$ as the result of the following operations on $G$.

- Replace the two subpaths $w_{1}^{\ell_{\text {pump }}}$ by $w_{1}^{x}$, where the number $x$ is chosen such that $x\left|w_{1}\right| \geq$ $2 t\left(n^{\prime}\right)+r$, and Type $\left(w_{1}^{\ell_{\text {pump }}}\right)=\operatorname{Type}\left(w_{1}^{x}\right)$.

- Replace the two subpaths $w_{2}^{\ell_{\text {pump }}}$ by $w_{2}^{y}$, where the number $y$ is chosen such that $y\left|w_{2}\right| \geq$ $2 t\left(n^{\prime}\right)+r$, and Type $\left(w_{2}^{\ell_{\text {pump }}}\right)=\operatorname{Type}\left(w_{2}^{y}\right)$.

The existence of the numbers $x$ and $y$ above is guaranteed by Lemma 15 . The IDs of nodes in $G^{\prime}$ are assigned as follows. For $i=1,2$, select the IDs of the nodes in $\bigcup_{v \in P_{i}^{\text {mid }}} N^{t\left(n^{\prime}\right)}(v)$ in such a way that the output labeling of $P_{i}^{\text {mid }}$ resulting from executing $\mathcal{A}$ on $G^{\prime}$ while assuming that the total number of nodes is $n^{\prime}$ is $f\left(w_{i}\right)^{2 r+1}$. The existence of such an ID assignment is guaranteed by Lemma 24 . For all remaining nodes in $G^{\prime}$, select their IDs in such a way that all nodes in $N^{r+t\left(n^{\prime}\right)}(v)$ receive distinct IDs, for each $v \in V\left(G^{\prime}\right)$. This ensures that the outcome of executing $\mathcal{A}$ on $G^{\prime}$ while assuming that the total number of nodes is $n^{\prime}$ is a legal labeling.

Let $\mathcal{L}_{\diamond}^{\prime}$ be the legal labeling of $G^{\prime}$ resulting from executing $\mathcal{A}$ with the above IDs while pretending that the total number of nodes is $n^{\prime}$. Note that $\mathcal{L}_{\diamond}^{\prime}$ must label $P_{1}^{\text {mid }}$ and $P_{2}^{\text {mid }}$ by $f\left(w_{1}\right)^{2 r+1}$ and $f\left(w_{2}\right)^{2 r+1}$, respectively. A desired legal labeling $\mathcal{L}_{\diamond}$ of $\mathcal{G}$ can be obtained from the legal labeling $\mathcal{L}_{\diamond}^{\prime}$ of $G^{\prime}$ by applying Lemma 11 , as we have Type $\left(w_{1}^{\ell_{\text {pump }}}\right)=\operatorname{Type}\left(w_{1}^{x}\right)$ and Type $\left(w_{2}^{\ell_{\text {pump }}}\right)=\operatorname{Type}\left(w_{2}^{y}\right)$.

\subsection{The $\omega(1)-o\left(\log ^{*} n\right)$ Gap}

In this section we prove that it is decidable whether a given $\mathrm{LCL}$ problem $\mathcal{P}$ has complexity $\Omega\left(\log ^{*} n\right)$ or $O(1)$ on cycle graphs. 
Lemma 26. Let $f$ be any feasible function. Let $G$ be any cycle graph. Let $\mathscr{P}$ be any set of disjoint subgraphs in $G$ such that the input labeling of each $P \in \mathscr{P}$ is of the form $w^{x}$ such that $x \geq 2 \ell_{\text {pump }}+2 r$, and $w \in \Sigma_{\text {in }}^{k}$ is a string with $1 \leq k \leq \ell_{\text {pump }}$. For each $P \in \mathscr{P}$, define the subgraph $P^{\prime}$ as follows. If $P$ is a cycle, define $P^{\prime}=P$. If $P$ is a path, write $P=w^{\ell_{\text {pump }}} \circ w^{i} \circ w^{\ell_{\text {pump }}}$, and define $P^{\prime}$ as the middle subpath $w^{i}$. Let $\mathcal{L}$ be a partial labeling of $G$ defined as follows. For each $P=w^{x} \in \mathscr{P}$, fix the output labels of each subpath $w$ of $P^{\prime}$ by $f(w)$. Then $\mathcal{G}=(G, \mathcal{L})$ admits a legal labeling $\mathcal{L}_{\diamond}$.

Proof. Define $V_{1}$ as the set of all nodes such that $v \in V_{1}$ if $v$ belongs to the middle subpath $w^{j}$ of some path $P=w^{\ell_{\text {pump }}} \circ w^{r} \circ w^{j} \circ w^{r} \circ w^{\ell_{\text {pump }}} \in \mathscr{P}$. By the definition of feasible function, $\mathcal{L}$ is already locally consistent at all nodes in $V_{1}$. Thus, all we need to do is to construct a complete labeling $\mathcal{L}_{\diamond}$ of $\mathcal{G}=(G, \mathcal{L})$, and argue that $\mathcal{L}_{\diamond}$ is locally consistent at all nodes in $V_{2}=V(G) \backslash V_{1}$.

There are two easy special cases. If $\mathscr{P}=\emptyset$, then no output label of any node in $G$ is fixed, and so $\mathcal{G}$ trivially admits a legal labeling. If $\mathscr{P}$ contains a cycle, then $\mathscr{P}=\{G\}$, and hence $\mathcal{L}$ is already a legal labeling as $V_{1}=V(G)$.

In subsequent discussion, we restrict ourselves to the case that $\mathscr{P}$ is non-empty and contains only paths. The output labeling $\mathcal{L}_{\diamond}$ is constructed as follows. Define $\mathscr{P}_{\text {unlabeled }}$ as the maximal-length subpaths of $G$ that are not assigned any output labels by $\mathcal{L}$. A path $P \in \mathscr{P}$ unlabeled must be of the form $w_{1}^{\ell_{\text {pump }}} \circ S \circ w_{2}^{\ell_{\text {pump }}}$, where $w_{1}, w_{2} \in \Sigma_{\text {in }}^{*}$ are two strings of length at least 1 and at most $\ell_{\text {pump }}$, and $S \in \Sigma_{\text {in }}^{*}$ can be any string (including the empty string). Given $P \in \mathscr{P}_{\text {unlabeled, we make the }}$ following definitions.

- Define $P^{+}$as the subpath of $G$ that includes $P$ and the $r\left|w_{1}\right|$ nodes preceding $P$, and the $r\left|w_{2}\right|$ nodes following $P$ in the graph $G$. Note that the set $V_{2}$ is exactly the union of nodes in $P^{+}$for all $P \in \mathscr{P}_{\text {unlabeled. }}$.

- Define $P^{++}$as the subpath of $G$ that includes $P$ and the $2 r\left|w_{1}\right|$ nodes preceding $P$, and the $2 r\left|w_{2}\right|$ nodes following $P$ in the graph $G$. The path $P^{++}$must be of the form $w_{1}^{\ell_{\text {pump }}+2 r} \circ S \circ$ $w_{2}^{\ell_{\text {pump }}+2 r}$, and the labeling $\mathcal{L}$ already fixes the output labels of the first $2 r\left|w_{1}\right|$ and the last $2 r\left|w_{2}\right|$ nodes of $P^{++}$by $f\left(w_{1}\right)^{2 r}$ and $f\left(w_{2}\right)^{2 r}$, respectively.

Observe that the path $P^{++}=w_{1}^{\ell_{\text {pump }}+2 r} \circ S \circ w_{2}^{\ell_{\text {pump }}+2 r}$ together with the labeling $\mathcal{L}$ is exactly the partially labeled graph $\mathcal{G}_{w_{1}, w_{2}, S}$. We assign the output labels to the nodes in $P$ by the labeling $\mathcal{L}_{\diamond}$ guaranteed in the definition of feasible function. It is ensured that the labeling of all nodes

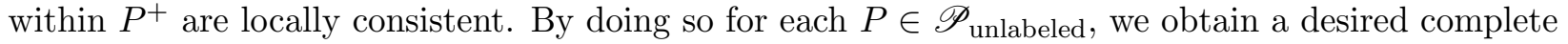
labeling that is locally consistent at all nodes in $V_{2}$.

Lemma 27. Suppose that there is a feasible function $f$ for the $\mathrm{LCL}$ problem $\mathcal{P}$. Then there is an $O(1)$-round deterministic LOCAL algorithm $\mathcal{A}$ on cycle graphs.

Proof. The first step of the algorithm $\mathcal{A}$ is to compute an $\left(\ell_{\text {width }}, \ell_{\text {count }}, \ell_{\text {pattern }}\right)$-partition in $O(1)$ rounds by Lemma 22 . We set $\ell_{\text {count }}=2 \ell_{\text {pump }}+2 r$ and $\ell_{\text {width }}=\ell_{\text {pattern }}=\ell_{\text {pump. }}$. We assume $|V(G)|>2 \ell_{\text {width }}$. Recall that an $\left(\ell_{\text {width }}, \ell_{\text {count }}, \ell_{\text {pattern }}\right)$-partition decomposes the cycle $G$ into two sets of disjoint subgraphs $\mathscr{P}_{\text {short }}$ and $\mathscr{P}_{\text {long }}$.

Define $G^{\prime}$ as the graph resulting from applying the following operations on $G$. For each $P \in \mathscr{P}_{\text {short }}$, replace the path $P$ by the path $P^{*}=x \circ y^{i} \circ z$ such that $i=\ell_{\text {count }}, 1 \leq|y| \leq \ell_{\text {pattern }}$, and the type of $P^{*}$ is the same as the type of $P$. The path $P^{*}$ is obtained via Lemma 14 . Note that each path $P \in \mathscr{P}_{\text {short }}$ has at least $\ell_{\text {width }}=\ell_{\text {pump }}$ nodes and at most $2 \ell_{\text {width }}=2 \ell_{\text {pump }}$ nodes. Define $\mathscr{P}^{*}$ as the set of all $P^{*}$ such that $P \in \mathscr{P}_{\text {short }}$. The graph $G^{\prime}$ is simulated in the communication graph $G$ by electing a leader for each path $P \in \mathscr{P}_{\text {short }}$ to simulate $P^{*}$. 


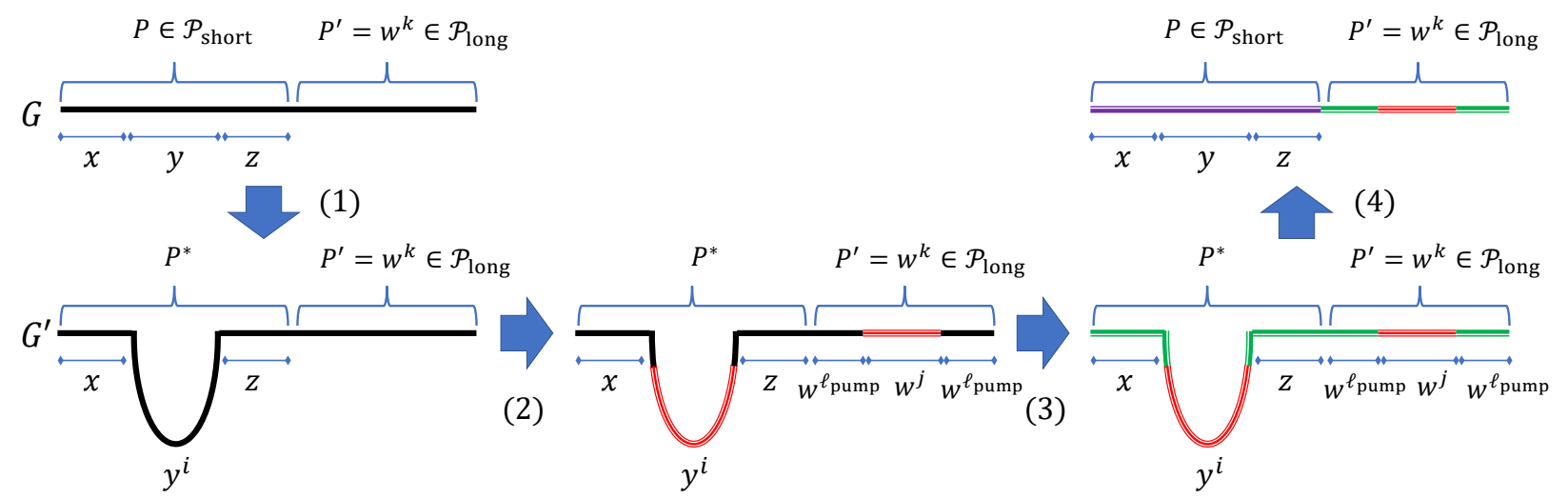

Figure 5: Illustration of Lemma 27.

Calculate a partial labeling $\mathcal{L}^{\prime}$ of $G^{\prime}$ using the feasible function $f$ as follows. Recall $\ell_{\text {count }}=$ $2 \ell_{\text {pump }}+2 r$. For each $P^{*}=x \circ y^{\ell_{\text {pump }}} \circ y^{2 r} \circ y^{\ell_{\text {pump }}} \circ z \in \mathscr{P}^{*}$, label the middle subpath $y^{2 r}$ by the function $f$. For each $P=w^{\ell_{\text {pump }}} \circ w^{i} \circ w^{\ell_{\text {pump }}} \in \mathscr{P}_{\text {long }}$, label the middle subpath $w^{i}$ by $f(w)^{i}$. Even though a path $P \in \mathscr{P}_{\text {long }}$ can have $\omega(1)$ nodes, this step can be done locally in $O(1)$ rounds due to the following property of $\left(\ell_{\text {width }}, \ell_{\text {count }}, \ell_{\text {pattern }}\right)$-partition. All nodes in a path $P \in \mathscr{P}$ long agree with the same direction and know the primitive string $w$.

By Lemma 26, the remaining unlabeled nodes in $G^{\prime}$ can be labeled to yield a legal labeling of $G^{\prime}$. This can be done in $O(1)$ rounds since the connected components formed by unlabeled nodes have at most $O(1)$ nodes. Given any valid labeling of $G^{\prime}$, a legal labeling of $G$ can be obtained by applying Lemma 11 in $O(1)$ rounds. Remember that Type $(P)=\operatorname{Type}\left(P^{*}\right)$ for each $P \in \mathscr{P}$ short, and $G^{\prime}$ is exactly the result of replacing each $P \in \mathscr{P}_{\text {short }}$ by $P^{*}$.

See Figure 5 for an illustration of Lemma 27: (1) applying a pumping lemma to extend each path $P \in \mathscr{P}_{\text {short }}$; (2) labeling the middle subpath $y^{2 r}$ of $P^{*}=x \circ y^{\ell_{\text {pump }}} \circ y^{2 r} \circ y^{\ell_{\text {pump }}} \circ z \in \mathscr{P}^{*}$ and the middle subpath $w^{j}$ of $P^{\prime}=w^{\ell_{\text {pump }}} \circ w^{i} \circ w^{\ell_{\text {pump }}} \in \mathscr{P}_{\text {long }}$ by the function $f$; (3) the remaining unlabeled nodes in $G^{\prime}$ can be labeled to yield a legal labeling of $G^{\prime}$ by Lemma 26; (4) since Type $(P)=\operatorname{Type}\left(P^{*}\right)$ for each $P \in \mathscr{P}_{\text {short }}$, we can recover a legal labeling of $G$ by re-labeling nodes in each $P \in \mathscr{P}_{\text {short }}$.

Combining Lemma 23, Lemma 25, and Lemma 27, we have proved Theorem 9. That is, for any $\mathrm{LCL}$ problem $\mathcal{P}$ on cycle graphs, its deterministic LOCAL complexity is either $\Omega\left(\log ^{*} n\right)$ or $O(1)$. Moreover, there is an algorithm that decides whether $\mathcal{P}$ has complexity $\Omega\left(\log ^{*} n\right)$ or $O(1)$ on cycle graphs; for the case the complexity is $O(1)$, the algorithm outputs a description of an $O(1)$-round deterministic LOCAL algorithm that solves $\mathcal{P}$.

\section{Acknowledgments}

Many thanks to Laurent Feuilloley, Juho Hirvonen, Janne H. Korhonen, Christoph Lenzen, Yannic Maus, and Seth Pettie for discussions, and to anonymous reviewers for their helpful comments on previous versions of this work. This work was supported in part by the Academy of Finland, Grant 285721. 


\section{References}

[1] Alkida Balliu, Sebastian Brandt, Dennis Olivetti, and Jukka Suomela. Almost global problems in the LOCAL model. In Proc. 32nd International Symposium on Distributed Computing (DISC 2018), Leibniz International Proceedings in Informatics (LIPIcs). Schloss DagstuhlLeibnizZentrum für Informatik, 2018. doi:10.4230/LIPIcs.DISC.2018.9.

[2] Alkida Balliu, Juho Hirvonen, Janne H Korhonen, Tuomo Lempiäinen, Dennis Olivetti, and Jukka Suomela. New classes of distributed time complexity. In Proc. 50th ACM Symposium on Theory of Computing (STOC 2018), pages 1307-1318. ACM Press, 2018. doi:10.1145/3188745. 3188860 .

[3] Roderick Bloem, Nicolas Braud-Santoni, and Swen Jacobs. Synthesis of Self-Stabilising and Byzantine-Resilient Distributed Systems. In Proc. International Conference on Computer Aided Verification (CAV 2016), pages 157-176. Springer, 2016. doi:10.1007/978-3-319-41528-4_9.

[4] Sebastian Brandt, Orr Fischer, Juho Hirvonen, Barbara Keller, Tuomo Lempiäinen, Joel Rybicki, Jukka Suomela, and Jara Uitto. A lower bound for the distributed Lovász local lemma. In Proc. 48th ACM Symposium on Theory of Computing (STOC 2016), pages 479-488. ACM Press, 2016. doi:10.1145/2897518.2897570.

[5] Sebastian Brandt, Juho Hirvonen, Janne H Korhonen, Tuomo Lempiäinen, Patric R J Östergård, Christopher Purcell, Joel Rybicki, Jukka Suomela, and Przemysław Uznański. LCL problems on grids. In Proc. 36th ACM Symposium on Principles of Distributed Computing (PODC 2017), pages 101-110. ACM Press, 2017. doi:10.1145/3087801.3087833.

[6] Yi-Jun Chang and Seth Pettie. A Time Hierarchy Theorem for the LOCAL Model. In Proc. 58th IEEE Symposium on Foundations of Computer Science (FOCS 2017), pages 156-167. IEEE, 2017. doi:10.1109/FOCS.2017.23.

[7] Yi-Jun Chang, Tsvi Kopelowitz, and Seth Pettie. An Exponential Separation between Randomized and Deterministic Complexity in the LOCAL Model. In Proc. 57th IEEE Symposium on Foundations of Computer Science (FOCS 2016), pages 615-624. IEEE, 2016. doi:10.1109/FOCS.2016.72.

[8] Richard Cole and Uzi Vishkin. Deterministic coin tossing with applications to optimal parallel list ranking. Information and Control, 70(1):32-53, 1986. doi:10.1016/S0019-9958(86)80023-7.

[9] Danny Dolev, Keijo Heljanko, Matti Järvisalo, Janne H Korhonen, Christoph Lenzen, Joel Rybicki, Jukka Suomela, and Siert Wieringa. Synchronous counting and computational algorithm design. Journal of Computer and System Sciences, 82(2):310-332, 2016. doi:10.1016/j. jcss.2015.09.002.

[10] Javier Esparza. Decidability and complexity of Petri net problems - An introduction. In Lectures on Petri Nets I: Basic Models: Advances in Petri Nets, pages 374-428. Springer Berlin Heidelberg, 1998. doi:10.1007/3-540-65306-6_20.

[11] Fathiyeh Faghih and Borzoo Bonakdarpour. SMT-Based Synthesis of Distributed Self-Stabilizing Systems. ACM Transactions on Autonomous and Adaptive Systems, 10(3):1-26, 2015. doi:10. $1145 / 2767133$. 
[12] Manuela Fischer and Mohsen Ghaffari. Sublogarithmic Distributed Algorithms for Lovász Local Lemma, and the Complexity Hierarchy. In Proc. 31st International Symposium on Distributed Computing (DISC 2017), pages 18:1-18:16, 2017. doi:10.4230/LIPIcs.DISC.2017.18.

[13] Mohsen Ghaffari and Hsin-Hao Su. Distributed Degree Splitting, Edge Coloring, and Orientations. In Proc. 28th ACM-SIAM Symposium on Discrete Algorithms (SODA 2017), pages 2505-2523. Society for Industrial and Applied Mathematics, 2017. doi:10.1137/1.9781611974782.166.

[14] Mohsen Ghaffari, David G Harris, and Fabian Kuhn. On Derandomizing Local Distributed Algorithms. In Proc. 59th IEEE Symposium on Foundations of Computer Science (FOCS 2018), 2018. doi:10.1109/FOCS.2018.00069.

[15] Mohsen Ghaffari, Juho Hirvonen, Fabian Kuhn, and Yannic Maus. Improved Distributed $\Delta$-Coloring. In Proc. 37th ACM Symposium on Principles of Distributed Computing (PODC 2018), pages 427-436. ACM, 2018. doi:10.1145/3212734.3212764.

[16] Andrew V. Goldberg, Serge A. Plotkin, and Gregory E. Shannon. Parallel Symmetry-Breaking in Sparse Graphs. SIAM Journal on Discrete Mathematics, 1(4):434-446, 1988. doi:10.1137/0401044.

[17] Juho Hirvonen, Joel Rybicki, Stefan Schmid, and Jukka Suomela. Large cuts with local algorithms on triangle-free graphs. Electronic Journal of Combinatorics, 24(4), 2017. URL http://www.combinatorics.org/ojs/index.php/eljc/article/view/v24i4p21.

[18] John E Hopcroft and Jeffrey D Ullman. Introduction to Automata Theory, Languages and Computation. Addison-Wesley, 1979.

[19] Alex Klinkhamer. On the Limits and Practice of Automatically Designing Self-Stabilization. Doctoral thesis, Michigan Technological University, 2016. URL https://digitalcommons.mtu. edu/etdr/90.

[20] Nathan Linial. Locality in Distributed Graph Algorithms. SIAM Journal on Computing, 21(1): 193-201, 1992. doi:10.1137/0221015.

[21] Moni Naor. A lower bound on probabilistic algorithms for distributive ring coloring. SIAM Journal on Discrete Mathematics, 4(3):409-412, 1991. doi:10.1137/0404036.

[22] Moni Naor and Larry Stockmeyer. What Can be Computed Locally? SIAM Journal on Computing, 24(6):1259-1277, 1995. doi:10.1137/S0097539793254571.

[23] Alessandro Panconesi and Aravind Srinivasan. The local nature of $\Delta$-coloring and its algorithmic applications. Combinatorica, 15(2):255-280, 1995. doi:10.1007/BF01200759.

[24] David Peleg. Distributed Computing: A Locality-Sensitive Approach. Society for Industrial and Applied Mathematics, 2000. doi:10.1137/1.9780898719772.

[25] Seth Pettie. Automatically Speeding Up LOCAL Graph Algorithms. In 7th Workshop on Advances in Distributed Graph Algorithms (ADGA 2018), 2018. URL http://adga.hiit.fi/2018/ Seth.pdf.

[26] Joel Rybicki and Jukka Suomela. Exact bounds for distributed graph colouring. In Proc. 22nd International Colloquium on Structural Information and Communication Complexity (SIROCCO 2015), volume 9439 of Lecture Notes in Computer Science, pages 46-60. Springer, 2015. doi:10.1007/978-3-319-25258-2_4. 